\title{
Nitrogen Fractionation in Protoplanetary Disks from the H13CN/HC15N Ratio
}

\section{Citation}

Guzmán, V. V., K. I. Öberg, J. Huang, R. Loomis, and C. Qi. 2017. Nitrogen Fractionation in Protoplanetary Disks from the H13CN/HC15N Ratio. The Astrophysical Journal 836, no. 1.

\section{Permanent link}

http://nrs.harvard.edu/urn-3:HUL.InstRepos:41292895

\section{Terms of Use}

This article was downloaded from Harvard University's DASH repository, and is made available under the terms and conditions applicable to Open Access Policy Articles, as set forth at http:// nrs.harvard.edu/urn-3:HUL.InstRepos:dash.current.terms-of-use\#OAP

\section{Share Your Story}

The Harvard community has made this article openly available.

Please share how this access benefits you. Submit a story.

\section{Accessibility}


Draft VERSion JANUARY 27, 2017

Preprint typeset using $\mathrm{IAT}_{\mathrm{E}} \mathrm{X}$ style emulateapj v. 12/16/11

\title{
NITROGEN FRACTIONATION IN PROTOPLANETARY DISKS FROM THE $\mathrm{H}^{13} \mathrm{CN} / \mathrm{HC}^{15} \mathrm{~N}$ RATIO
}

\author{
V.V. Guzmán, K.I. Öberg, J. Huang, R. Loomis, and C. Qi \\ Harvard-Smithsonian Center for Astrophysics, 60 Garden Street, Cambridge, MA 02138, USA \\ Draft version January 27, 2017
}

\begin{abstract}
Nitrogen fractionation is commonly used to assess the thermal history of Solar System volatiles. With ALMA it is for the first time possible to directly measure ${ }^{14} \mathrm{~N} /{ }^{15} \mathrm{~N}$ ratios in common molecules during assembly of planetary systems. We present ALMA observations of the $\mathrm{H}^{13} \mathrm{CN}$ and $\mathrm{HC}^{15} \mathrm{~N} \mathrm{~J}=3-2$ lines at $0^{\prime \prime} .5$ angular resolution, toward a sample of six protoplanetary disks, selected to span a range of stellar and disk structure properties. Adopting a typical ${ }^{12} \mathrm{C} /{ }^{13} \mathrm{C}$ ratio of 70 , we find comet-like ${ }^{14} \mathrm{~N} /{ }^{15} \mathrm{~N}$ ratios of $80-160$ in $5 / 6$ of the disks (3 T Tauri and 2 Herbig Ae disks) and lack constraints for one of the T Tauri disks (IM Lup). There are no systematic differences between $\mathrm{T}$ Tauri and Herbig Ae disks, or between full and transition disks within the sample. In addition, no correlation is observed between disk-averaged $\mathrm{D} / \mathrm{H}$ and ${ }^{14} \mathrm{~N} /{ }^{15} \mathrm{~N}$ ratios in the sample. One of the disks, V4046 Sgr, presents unusually bright HCN isotopologue emission, enabling us to model the radial profiles of $\mathrm{H}^{13} \mathrm{CN}$ and $\mathrm{HC}^{15} \mathrm{~N}$. We find tentative evidence of an increasing ${ }^{14} \mathrm{~N} /{ }^{15} \mathrm{~N}$ ratio with radius, indicating that selective photodissociation in the inner disk is important in setting the ${ }^{14} \mathrm{~N} /{ }^{15} \mathrm{~N}$ ratio during planet formation.
\end{abstract}

Keywords: astrochemistry ISM: clouds ISM: molecules radiative transfer radio lines: ISM

\section{INTRODUCTION}

The origin of Solar System organics is a fundamental and highly debated topic. It is unclear whether the organics in the different Solar System bodies were inherited from the cold and dense molecular parent cloud of our Sun, or if they are the result of chemical processing within the Solar nebula protoplanetary disk. The isotopic composition of present day organics may help to shed light on their origins, since isotopic fractionation chemistry is highly environment specific and can leave a permanent imprint. Comets are especially interesting in this context, since they should preserve the isotopic compositions in different molecules during the assembly of the Solar System.

Among the different methods used to trace the origin of molecules, the ${ }^{14} \mathrm{~N} /{ }^{15} \mathrm{~N}$ isotopic ratio is one of the most popular ones. Nitrogen isotopic ratios span at least an order of magnitude between different Solar System bodies. A low nitrogen fractionation (high ${ }^{14} \mathrm{~N} /{ }^{15} \mathrm{~N}$ ) is observed toward the Sun and Jupiter $\left({ }^{14} \mathrm{~N} /{ }^{15} \mathrm{~N}=450\right.$; Marty et al. 2011), while a high fractionation is observed in the rocky planets, comets and meteorites $\left({ }^{14} \mathrm{~N} /{ }^{15} \mathrm{~N}=\right.$ 100-300; Mumma \& Charnley 2011). The origin of these ${ }^{14} \mathrm{~N} /{ }^{15} \mathrm{~N}$ variations is not well understood, but it suggests that different Solar System bodies obtained their nitrogen from different nitrogen reservoirs (Mumma \& Charnley 2011). Based on observations of the Interstellar Medium (ISM), comets and chemical models, there are three major nitrogen reservoirs in dense interstellar and circumstellar media, $\mathrm{N}_{2}, \mathrm{NH}_{3}$ and $\mathrm{HCN}$. These species have different fractionation pathways (e.g., HilyBlant et al. 2013), and it is thus important to measure

vguzman@cfa.harvard.edu

* Currently at Joint Atacama Large Millimeter/submillimeter Array (ALMA) Observatory, Avenida Alonso de Córdova 3107, Vitacura, Santiago, Chile. the ${ }^{14} \mathrm{~N} /{ }^{15} \mathrm{~N}$ ratio in molecules representative of these nitrogen reservoirs.

This study focuses on $\mathrm{HCN}$. HCN is readily detected in the ISM (e.g., Liszt \& Lucas 2001; Hily-Blant et al. 2013), comets (e.g., Bockelée-Morvan et al. 2008) and protoplanetary disks (e.g., Thi et al. 2004 Oberg et al. 2010, 2011; Chapillon et al. 2012). While several studies have been made toward prestellar cores and protostars, the characterization of isotopic ratios in protoplanetary disks is rather new due to the intrinsic weak line emission. Guzmán et al. (2015) presented the first detection of $\mathrm{H}^{13} \mathrm{CN}$ and $\mathrm{HC}^{15} \mathrm{~N}$ in the disk around Herbig $\mathrm{Ae}$ star MWC 480, and provided the first measurement of the ${ }^{14} \mathrm{~N} /{ }^{15} \mathrm{~N}$ in a disk. They found an isotopic ratio of $200 \pm 100$, which is similar to what is observed in the cold ISM and in comets.

The low ${ }^{14} \mathrm{~N} /{ }^{15} \mathrm{~N}$ value in the MWC 480 disk implies either inheritance of HCN from the ISM, or the presence of an active fractionation chemistry in the disk. There are two potentially active fractionation channels in disks and in the ISM. The first is through isotope exchange reactions, such as

$$
\mathrm{HC}^{14} \mathrm{NH}^{+}+{ }^{15} \mathrm{~N} \rightarrow \mathrm{HC}^{15} \mathrm{NH}^{+}+{ }^{14} \mathrm{~N}+h \nu
$$

which favor the incorporation of ${ }^{15} \mathrm{~N}$ into molecules at low temperatures $(<20 \mathrm{~K})$. $\mathrm{HC}^{15} \mathrm{NH}^{+}$can later recombine with free electrons to produce $\mathrm{HC}^{15} \mathrm{~N}$. Observations of $\mathrm{HCN}$ and $\mathrm{HNC}$ fractionation toward protostars present a tentative trend of the ${ }^{14} \mathrm{~N} /{ }^{15} \mathrm{~N}$ with temperature, supporting this scenario (Wampfler et al. 2014). The second mechanism is selective photo-dissociation of ${ }^{14} \mathrm{~N}^{15} \mathrm{~N}$ over ${ }^{14} \mathrm{~N}_{2}$, due to self-shielding of ${ }^{14} \mathrm{~N}_{2}$ (Heays et al. 2014). In the surface layers of protoplanetary disks, which are directly illuminated by the radiation field of the central star, the dominant formation pathways lead- 
Table 1: Line observations.

\begin{tabular}{|c|c|c|c|c|c|c|c|c|}
\hline \multirow[b]{2}{*}{ Source } & \multicolumn{4}{|c|}{$\mathrm{H}^{13} \mathrm{CN} J=3-2$} & \multicolumn{4}{|c|}{$\mathrm{HC}^{15} \mathrm{~N} J=3-2$} \\
\hline & $\begin{array}{l}\text { Beam } \\
\text { arcsec }\end{array}$ & PA & $\begin{array}{l}\text { Channel } \mathrm{rms}^{a} \\
\text { mJy beam } \\
-1\end{array}$ & $\begin{array}{c}\text { Mom. zero } \mathrm{rms}^{a} \\
\mathrm{mJy} \mathrm{km} \mathrm{s} \mathrm{s}^{-1}\end{array}$ & $\begin{array}{l}\text { Beam } \\
\text { arcsec }\end{array}$ & $\mathrm{PA}$ & $\begin{array}{l}\text { Channel } \mathrm{rms}^{a} \\
\text { mJy beam }\end{array}$ & $\begin{array}{l}\text { Mom. zero rms } \\
\text { mJy km s} ~^{-1}\end{array}$ \\
\hline AS 209 & $0.51 \times 0.50$ & -86.9 & 4.3 & 7.6 & $0.51 \times 0.44$ & -62.4 & 3.4 & 6.5 \\
\hline IM Lup & $0.46 \times 0.43$ & 71.1 & 3.4 & 5.6 & $0.47 \times 0.43$ & 71.8 & 3.0 & 5.2 \\
\hline $\mathrm{LkCa} 15$ & $0.65 \times 0.47$ & -13.0 & 3.2 & 6.3 & $0.67 \times 0.50$ & -14.7 & 2.9 & 5.7 \\
\hline V4046 Sgr & $0.60 \times 0.48$ & 87.3 & 3.4 & 10.4 & $0.59 \times 0.48$ & 87.0 & 3.2 & 9.4 \\
\hline MWC 480 & $0.74 \times 0.46$ & -8.7 & 3.3 & 7.0 & $0.74 \times 0.46$ & -9.0 & 3.1 & 6.7 \\
\hline HD 163296 & $0.57 \times 0.44$ & -87.9 & 2.6 & 6.9 & $0.59 \times 0.46$ & -87.3 & 2.4 & 6.4 \\
\hline
\end{tabular}

${ }^{a}$ Channel rms noise in $0.5 \mathrm{~km} \mathrm{~s}^{-1}$ channel width.

Table 2: Star and disk properties in the sample.

\begin{tabular}{|c|c|c|c|c|c|c|c|c|c|c|c|}
\hline Source & $\begin{array}{c}\text { Distance } \\
\text { pc }\end{array}$ & $\begin{array}{c}\text { Spectral } \\
\text { type }\end{array}$ & $\begin{array}{l}\text { Age } \\
\text { Myr }\end{array}$ & $\begin{array}{l}\mathrm{M}_{\star} \\
\mathrm{M}_{\odot}\end{array}$ & $\begin{array}{l}\mathrm{L}_{\star} \\
\mathrm{L}_{\odot}\end{array}$ & $10^{-9}$ & $\begin{array}{l}\dot{\mathrm{M}}_{\star} \\
\mathrm{M}_{\odot \mathrm{yr}^{-1}}\end{array}$ & $\begin{array}{l}\text { Disk Incl. } \\
\quad \text { deg }\end{array}$ & $\begin{array}{c}\text { Disk PA } \\
\text { deg }\end{array}$ & $\begin{array}{c}\mathrm{M}_{\text {disk }} \\
\mathrm{M}_{\odot}\end{array}$ & $\begin{array}{c}v_{L S R} \\
\mathrm{~km} \mathrm{~s}^{-1}\end{array}$ \\
\hline \multicolumn{12}{|c|}{ T Tauri } \\
\hline AS 209 & 126 & K5 & 1.6 & 0.9 & 1.5 & & 51 & 38 & 86 & 0.015 & 4.6 \\
\hline IM Lup & 161 & M0 & 1 & 1.0 & 0.93 & & 0.01 & 50 & 144.5 & 0.17 & 4.4 \\
\hline LkCa 15 & 140 & K3 & $3-5$ & 0.97 & 0.74 & & 1.3 & 52 & 60 & $0.05-0.1$ & 6.3 \\
\hline V4046 Sgr & 72 & $\mathrm{~K} 5, \mathrm{~K} 7$ & 24 & 1.75 & $0.49,0.33$ & & 0.5 & 33.5 & 76 & 0.028 & 2.9 \\
\hline \multicolumn{12}{|c|}{ Herbig Ae } \\
\hline MWC 480 & 142 & A4 & 7 & 1.65 & 11.5 & & 126 & 37 & 148 & 0.11 & 5.1 \\
\hline HD 163296 & 122 & $\mathrm{~A} 1$ & 4 & 2.25 & 30 & & 69 & 48.5 & 132 & 0.17 & 5.8 \\
\hline
\end{tabular}

Note: Table reproduced from Huang et al. (2017), where a complete list of references is given.

ing to $\mathrm{HCN}$ and $\mathrm{HC}^{15} \mathrm{~N}$ are

$$
\begin{aligned}
& { }^{14} \mathrm{~N}+\mathrm{CH}_{2} \rightarrow \mathrm{HC}^{14} \mathrm{~N} \\
& { }^{15} \mathrm{~N}+\mathrm{CH}_{2} \rightarrow \mathrm{HC}^{15} \mathrm{~N}
\end{aligned}
$$

Both mechanisms can reduce the $\mathrm{HCN} / \mathrm{HC}^{15} \mathrm{~N}$ ratio in protoplanetary disks (Lyons et al. 2009. Heays et al. 2014). Distinguishing between these different origins of nitrogen fractionation levels in disks and between inheritance and in situ disk fractionation chemistry (and further in comets and planets) requires more disk measurements, and constraints on the radial profiles of the ${ }^{14} \mathrm{~N} /{ }^{15} \mathrm{~N}$ ratio in disks with different structures and around stars with different radiation fields. A constant ${ }^{14} \mathrm{~N} /{ }^{15} \mathrm{~N}$ ratio across disks would favor a scenario where disks inherit their organics from the natal cloud, while disk chemistry should result in a radial gradient, since the disk environment is dramatically different at different radii.

To begin to address this long-term goal, we present observations of $\mathrm{H}^{13} \mathrm{CN}$ and $\mathrm{HC}^{15} \mathrm{~N}$ in a diverse sample of 6 protoplanetary disks. Because the $\mathrm{HCN}$ lines may be optically thick, we use the $\mathrm{H}^{13} \mathrm{CN}$ line as a proxy of $\mathrm{HCN}$ to derive the ${ }^{14} \mathrm{~N} /{ }^{15} \mathrm{~N}$ ratio. In section 2 we present the observations and describe the data reduction process. The disk-averaged isotopic flux ratios derived from the observations are presented in Section 3. In Section 4, we model the disk abundance profiles of $\mathrm{H}^{13} \mathrm{CN}$ and $\mathrm{HC}^{15} \mathrm{~N}$ in V4046 Sgr, the source with the highest signal-to-noise ratio detection. In Section 5, we discuss the results and compare with observations in our Solar System and in the cold ISM. A summary is presented in Section 6 .

\section{OBSERVATIONS AND DATA REDUCTION}

The $\mathrm{H}^{13} \mathrm{CN}$ and $\mathrm{HC}^{15} \mathrm{~N} J=3-2$ lines were observed with ALMA during Cycle 2 as part of project ADS/JAO.ALMA\#2013.1.00226. The Band 6 observations included two spectral settings, at 1.1 and $1.4 \mathrm{~mm}$.
The correlator setup of the $1.1 \mathrm{~mm}$ and $1.4 \mathrm{~mm}$ settings were configured with 14 and 13 narrow spectral windows, respectively, targeting different molecular lines. The main targets of the observations were deuterated species, which are presented in Huang et al. (2017). They include an independent analysis of the $\mathrm{H}^{13} \mathrm{CN}$ data in the context of $\mathrm{D} / \mathrm{H}$ fractionation. A focused study on the $\mathrm{DCO}^{+}$emission in IM Lup was presented earlier by Öberg et al. (2015). The $\mathrm{H}^{13} \mathrm{CN}$ data in MWC 480 was also used for the study of $\mathrm{CH}_{3} \mathrm{CN}$ by Öberg et al. (2015). In addition, Guzmán et al. (2015) presented the $\mathrm{H}^{13} \mathrm{CN}$ and $\mathrm{HC}^{15} \mathrm{~N}$ data in MWC 480. In this paper, we focus on the $\mathrm{H}^{13} \mathrm{CN}$ and $\mathrm{HC}^{15} \mathrm{~N}$ lines for the full sample. For consistency, we present data reduction and imaged independently of the previous papers. For one of the sources, V4046 Sgr, we also use CO $J=2-1$ isotopologue data from the same survey.

The observations are described in detail by Huang et al. (2017). In short, the Band 6 observations were carriedout between 2014 and 2015 with baseline lengths spanning between 18 and $650 \mathrm{~m}$. The total on-source time was $20 \mathrm{~min}$, on average. A quasar was observed to calibrate amplitude and phase temporal variations. To calibrate the frequency bandpass a quasar was observed. The absolute flux scale was derived by observing Titan for about half the observations, or a quasar. The HCN isotopologue lines were covered by two spectral windows of $59 \mathrm{MHz}$ bandwidth and $61 \mathrm{kHz}$ channel width in the $1.1 \mathrm{~mm}$ spectral setting. The CO isotopologue lines were covered by three spectral windows in the $1.4 \mathrm{~mm}$ spectral setting, with the same bandwidth and channel width.

The data calibration was performed by the ALMA staff using standard procedures. We took advantage of the bright continuum emission of the sources to improve the signal-to-noise ratio, by further self-calibrating the $\mathrm{HCN}$ isotopologue data. The self-calibration solutions were 

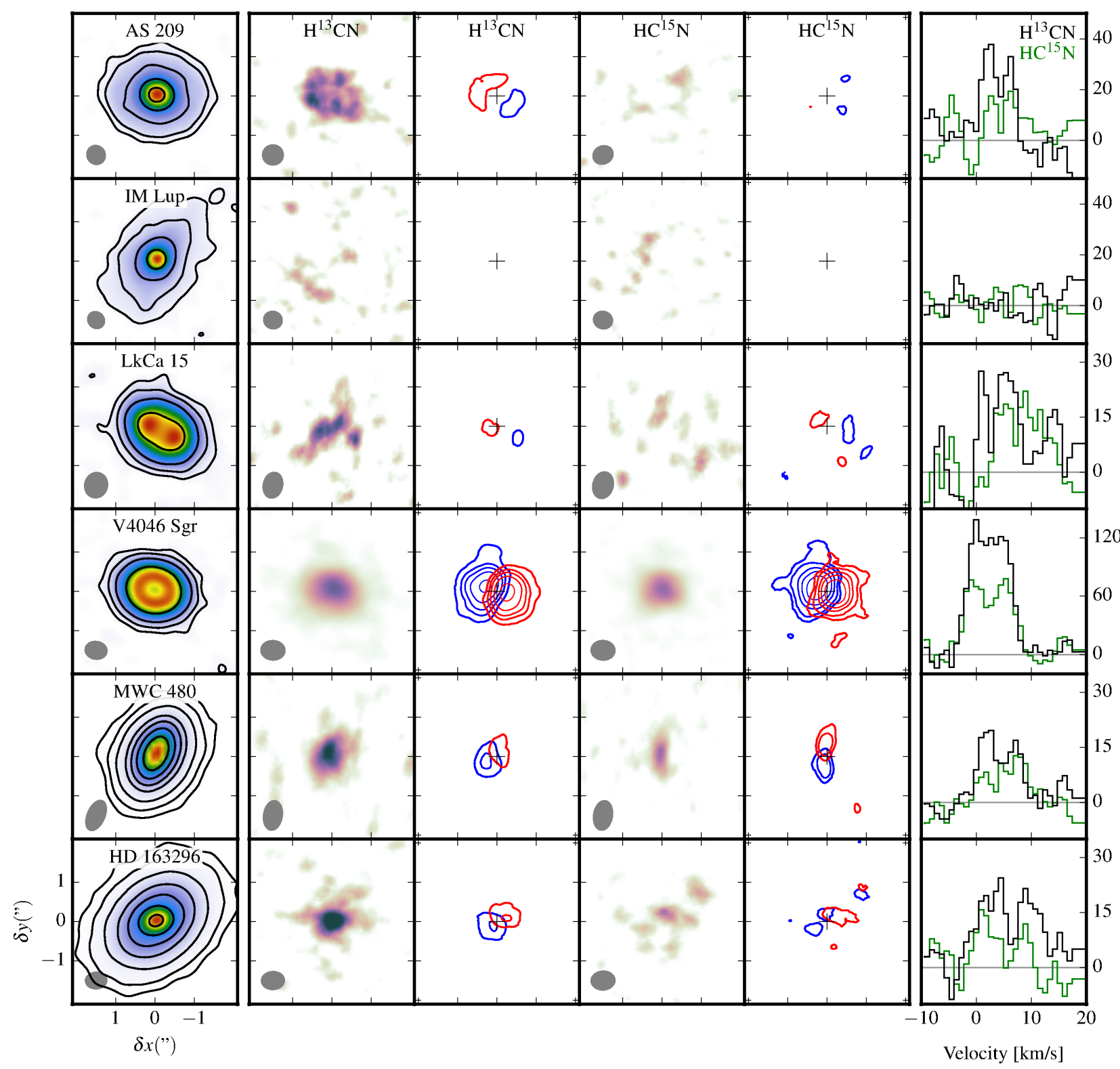

Figure 1. : Left: Dust continuum emission. Contour levels correspond to 3, 10, 30, 100, 200, 400 and 800 $\sigma$. Middle: Moment zero maps for $\mathrm{H}^{13} \mathrm{CN}$ and $\mathrm{HC}^{15} \mathrm{~N}$, integrated over the full line width (colors) and over the red- and blue-shifted parts of the line (contours). Noise below $2 \mathrm{mJy}_{\text {beam }}{ }^{-1}$ has been clipped to produce the moment zero maps. Color scales start at $2 \sigma$. Contour levels correspond to $3,5,7,10,15,20$ and $30 \sigma$. For $\mathrm{HC}^{15} \mathrm{~N}$ contour levels start at $2 \sigma$. Right: Disk integrated spectra, extracted using elliptical masks (see Appendix).

derived on individual spectral windows when possible (AS 209, LkCa 15, MWC 480 and V4046 Sgr) and on averaged spectral windows for the weaker sources (IM Lup and HD 163296), and then applied to each spectral window. The continuum was then subtracted from the visibilities to produce the spectral line cubes. The clean images were obtained by deconvolving the visibilities in CASA, using the CLEAN algorithm with Briggs weighting. The HCN isotopologue data was regridded to a spectral resolution of $0.5 \mathrm{~km} \mathrm{~s}^{-1}$ for the full sample. The robust parameter was set to 1.0 for $\mathrm{H}^{13} \mathrm{CN}$, except for IM Lup where we used a value of 2.0. For $\mathrm{HC}^{15} \mathrm{~N}$ the robust parameter was set to 2.0 to improve the signal- to-noise ratio, except for V4046 Sgr and MWC 480 for which a value of 1.0 was used because of the bright line emission. To help the cleaning process we created elliptical masks, same for all channels, centered on the dust continuum centroid, using the disk inclination and position angle listed in Table 2 . The mask radius was chosen to cover the stronger $\mathrm{H}^{13} \mathrm{CN}$ line emission in all channels, or to cover the dust continuum emission if the line was not detected. The resulting beam, channel rms and moment zero rms values are listed in Table 1. For the $\mathrm{CO}$ isotopologues the robust parameter was set to 0.5 , and a Keplerian mask was used to help the cleaning process, created by selecting emission consistent with the 
Table 3: Integrated fluxes and inferred abundance ratios in the disk sample.

\begin{tabular}{lrrcr}
\hline Source & $F\left(\mathrm{H}^{13} \mathrm{CN}\right)^{a}$ & $F\left(\mathrm{HC}^{15} \mathrm{~N}\right)^{a}$ & $N\left(\mathrm{H}^{13} \mathrm{CN}\right) / N\left(\mathrm{HC}^{15} \mathrm{~N}\right)^{b}$ & ${ }^{14} \mathrm{~N} /{ }^{15} \mathrm{~N}^{c}$ \\
\hline AS 209 & $175 \pm 25$ & $79 \pm 23$ & $2.2 \pm 0.7$ & $156 \pm 71$ \\
IM Lup & $<50^{d}$ & $<50^{d}$ & - & - \\
LkCa 15 & $120 \pm 20$ & $101 \pm 18$ & $1.2 \pm 0.3$ & $83 \pm 32$ \\
V4046 Sgr & $1098 \pm 35$ & $670 \pm 26$ & $1.6 \pm 0.1$ & $115 \pm 35$ \\
MWC 480 & $132 \pm 13$ & $75 \pm 13$ & $1.8 \pm 0.3$ & $123 \pm 45$ \\
HD 163296 & $177 \pm 23$ & $87 \pm 21$ & $2.0 \pm 0.6$ & $142 \pm 59$ \\
\hline
\end{tabular}

${ }^{a}$ The uncertainties do not include absolute flux calibration errors. ${ }^{b}$ Computed assuming LTE and $T_{\text {ex }}=15 \mathrm{~K} .{ }^{c}$ Adopting ${ }^{12} \mathrm{C} /{ }^{13} \mathrm{C}=70$ and an associated uncertainty of $30 \% .{ }^{d} 3 \sigma$ upper limits.

Table 4: Spectroscopic parameters.

\begin{tabular}{|c|c|c|c|c|}
\hline Line & $\begin{array}{c}\text { Frequency } \\
\text { GHz }\end{array}$ & $\begin{array}{c}E_{u} / k \\
\mathrm{~K}\end{array}$ & $\begin{array}{l}A_{u l} \\
\mathrm{~s}^{-1}\end{array}$ & $g_{u}$ \\
\hline $\mathrm{H}^{13} \mathrm{CN} J=3-2$ & 259.0118 & 24.86 & $7.7 \times 10^{-4}$ & 21 \\
\hline $\mathrm{HC}^{15} \mathrm{~N} J=3-2$ & 258.1571 & 24.78 & $7.6 \times 10^{-4}$ & 7 \\
\hline
\end{tabular}

expected Keplerian rotation of the disk in each channel.

\section{SAMPLE STATISTICS}

The stellar and disk properties of the sample are summarized in Table 2. The sample includes $4 \mathrm{~T}$ Tauri stars and 2 Herbig Ae stars. The stellar masses range between 0.9 (AS 209) and $2.3 \mathrm{M}_{\odot}(\mathrm{HD} 163296)$, corresponding to luminosities that span an order of magnitude. Two of the sources, namely LkCa 15 and V4046 Sgr, are transitional disks, with inner holes resolved at millimeter wavelengths. The sample is biased toward large disks, with a known rich molecular emission. However, given the very different physical properties, in particular the gas temperature, the source selection allows us to determine the disk averaged ${ }^{14} \mathrm{~N} /{ }^{15} \mathrm{~N}$ ratio in $\mathrm{HCN}$ in a diverse sample of disks.

Figure 1 shows the observations for the full sample of disks. The dust continuum images are shown in the left column. The $1.1 \mathrm{~mm}$ continuum images were produced by averaging $1.1 \mathrm{~mm}$ line free spectral windows. The four middle panels display the $\mathrm{H}^{13} \mathrm{CN}$ and $\mathrm{HC}^{15} \mathrm{~N}$ velocity integrated maps, for the full line (color images) and for two velocity ranges, the blue and red shifted parts of the line, to demonstrate the Keplerian rotation of the disk (blue and red contours). The right column of the figure shows the disk integrated line profiles of the $\mathrm{H}^{13} \mathrm{CN}$ and $\mathrm{HC}^{15} \mathrm{~N}$ lines. The spectra were extracted using the same elliptical masks used to clean the data. Figs. A.1 to A.6 show channels maps of the $\mathrm{H}^{13} \mathrm{CN}$ and $\mathrm{HC}^{15} \mathrm{~N}$ lines in each source, with the elliptical masks overlaid on top.

The lines are classified as detected if emission consistent with the expected Keplerian rotation of the disk is observed in at least three channels at a $3 \sigma$ level. From the inspection of the channel maps we find that $\mathrm{H}^{13} \mathrm{CN}$ is clearly detected toward all disks except for the $\mathrm{T}$ Tauri disk IM Lup. $\mathrm{HC}^{15} \mathrm{~N}$ is clearly detected toward V4046 Sgr, MWC 480 and HD 163296, weakly detected toward AS 209 and $\mathrm{LkCa}$ 15, and not detected toward IM Lup. We note that for the two disks with weak $\mathrm{HC}^{15} \mathrm{~N}$ line emission, while emission is detected in 3 or more individual channels, the emission is almost washed out in the integrated intensity maps.

The $\mathrm{H}^{13} \mathrm{CN}$ emission is generally compact compared to the extent of the dust disk. It appears centrally peaked toward MWC 480 and V4046 Sgr, and possibly toward
HD 163296, but presents clear rings toward the remaining two sources: LkCa 15 and AS 209. The latter is unexpected, since AS 209 is not a transition disk. The $\mathrm{HC}^{15} \mathrm{~N}$ line shows a similar behavior, although the signalto-noise ratio is lower.

We extracted the disk integrated fluxes from the unclipped moment zero maps using the same elliptical mask created to clean the data and extract the spectra. The uncertainty in the flux was estimated by simulating integrated flux measurements from signal free regions using the same elliptical mask but centered at random positions. The integrated disk fluxes and their associated uncertainties are listed in Table 3 .

We use the extracted line flux ratios to estimate the abundance ratios of the two isotopologues. This is a reasonable first approximation when comparing $\mathrm{HCN}$ isotopologes, as the $\mathrm{H}^{13} \mathrm{CN}$ and $\mathrm{HC}^{15} \mathrm{~N}$ line emission is expected to be optically thin, arise in the same region and this region is dense enough for the molecular rotational population to be in LTE (Pavlyuchenkov et al. 2007). We compute the $\mathrm{H}^{13} \mathrm{CN} / \mathrm{HC}^{15} \mathrm{~N}$ abundance ratio in the LTE case and for $T_{\text {ex }}=15 \mathrm{~K}$. For MWC 480, we obtain a lower $\mathrm{H}^{13} \mathrm{CN} / \mathrm{HC}^{15} \mathrm{~N}$ ratio $(1.8 \pm 0.3)$ than the value of $2.8 \pm 1.4$ reported in Guzmán et al. (2015), although both values are consistent within the errors. This is due to the different method implemented in this paper to extract the fluxes. The resulting $\mathrm{H}^{13} \mathrm{CN} / \mathrm{HC}^{15} \mathrm{~N}$ abundance ratios span from 1.2 to 2.2 with an average of 1.8. Given the almost identical upper energies, Einstein coefficients and the ratio $Q / g_{u}$ (partition function over upper state degeneracy) for the $J=3-2$ transition (see Table 4), the $\mathrm{H}^{13} \mathrm{CN} / \mathrm{HC}^{15} \mathrm{~N}$ abundance ratios are almost identical to the flux ratios. We note that a higher excitation temperature of $100 \mathrm{~K}$ changes the inferred abundances by less than $1 \%$.

In order to derive the nitrogen fractionation in $\mathrm{HCN}$, we adopt an isotopic ratio of ${ }^{12} \mathrm{C} /{ }^{13} \mathrm{C}=70$. Because the $\mathrm{C}$ isotopic ratio depends on the physical conditions of the gas (e.g., Roueff et al. 2015) we include a $30 \%$ uncertainty in this value to convert the $\mathrm{H}^{13} \mathrm{CN} / \mathrm{HC}^{15} \mathrm{~N}$ ratio into a ${ }^{14} \mathrm{~N} /{ }^{15} \mathrm{~N}$ ratio. The inferred ${ }^{14} \mathrm{~N} /{ }^{15} \mathrm{~N}$ ratios span from 83 to 156 with an average of 124 . All disks present low cometary-like ${ }^{14} \mathrm{~N} /{ }^{15} \mathrm{~N}$ ratios. The resulting $\mathrm{H}^{13} \mathrm{CN} / \mathrm{HC}^{15} \mathrm{~N}$ abundance ratios and the inferred ${ }^{14} \mathrm{~N} /{ }^{15} \mathrm{~N}$ flux ratios are listed in Table 3. Fig. 2 shows the nitrogen fractionation ratios for the full sample and compares it with $\mathrm{DCN} / \mathrm{HCN}$ ratios derived by Huang et al. (2017). There is no indication of a correlation between the disk averaged nitrogen and hydrogen fractionation in these disks, as might have been expected if both originated from a cold fractionation pathway (see also sec- 


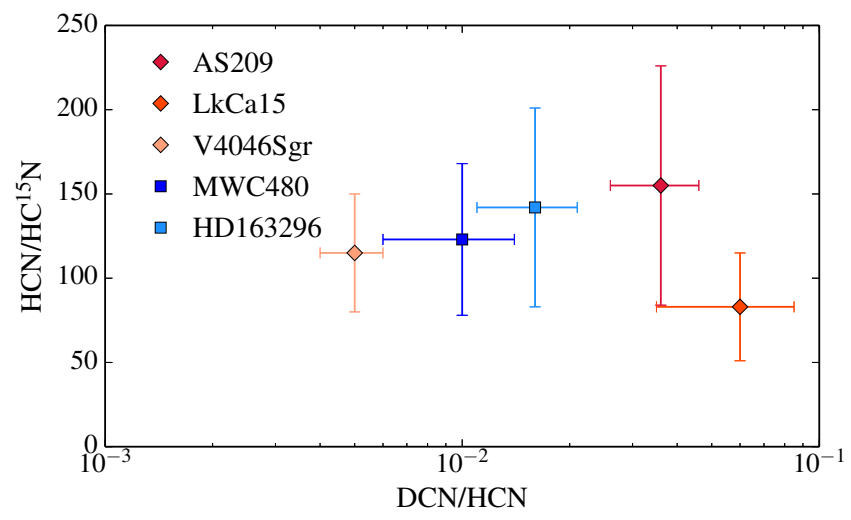

Figure 2. : $\mathrm{N}$ fractionation as a function of deuterium fractionation. Disks around T Tauri stars are shown in red colors, while disks around Herbig Ae stars are shown in blue colors. The $\mathrm{DCN} / \mathrm{HCN}$ ratios are taken from Huang et al. (2017). Both abundance ratios are computed assuming LTE conditions and $T_{\mathrm{ex}}=15 \mathrm{~K}$.

tion 5.3. We note that the spread in ${ }^{14} \mathrm{~N} /{ }^{15} \mathrm{~N}$ is small compared to the errors and we cannot rule out that there is a trend that is washed out by the noise.

\section{THE $\mathrm{H}^{13} \mathrm{CN} / \mathrm{HC}^{15} \mathrm{~N}$ PROFILE IN V4046 SGR}

HCN isotopologue emission observed toward V4046 Sgr is sufficiently bright to provide constraints on the $\mathrm{H}^{13} \mathrm{CN}$ and $\mathrm{HC}^{15} \mathrm{~N}$ abundance profiles. In this section, we model the emission profile of the observed lines in order to retrieve the underlying ${ }^{14} \mathrm{~N} /{ }^{15} \mathrm{~N}$ abundance ratio across the disk.

\subsection{Disk physical structure}

In order to investigate possible variations of the abundance ratio across the disk a detailed model of the line emission is needed. We build a parametric model to describe the physical structure of the disk based on the model described in Rosenfeld et al. (2013), which was constructed to reproduce the emission of the dust continuum and the $\mathrm{CO}$ isotopologues. We first parametrize the dust surface density as

$\Sigma_{\text {dust }}(r)= \begin{cases}\Sigma_{c}\left(\frac{r}{r_{c}}\right)^{-\gamma} \exp \left[-\left(\frac{r}{r_{c}}\right)^{2-\gamma}\right] & r \geq r_{c a v} \\ \Sigma_{c a v} & 0.2 \mathrm{AU}<\end{cases}$

where $\Sigma_{c}$ is a normalization factor, $r_{c}$ is a characteristic radius, $\gamma=1$ is the power-law index of the viscosity, and $r_{c a v}$ is the radius of the inner cavity. We include two dust populations, one for the atmosphere and another for the midplane grains. The dust volume density is computed assuming a vertical Gaussian distribution of each dust grain population:

$$
\rho_{\text {dust }}=\sum_{i=0,1} \frac{\Sigma_{i}(r)}{\sqrt{2 \pi} H_{i}(r)} \exp \left(\frac{-z^{2}}{H_{i}(r)^{2}}\right),
$$

The midplane grains, which are larger than the atmospheric ones, comprise the bulk of the dust mass $\left(\Sigma_{m i d}=0.9 \Sigma_{d u s t}, \Sigma_{a t m}=0.1 \Sigma_{d u s t}\right)$. The atmospheric dust grains are vertically Gaussian distributed with a scale height:

$$
H_{a t m}(r)=H_{10}\left(\frac{r}{100 \mathrm{AU}}\right)^{h}
$$

and the midplane dust grains are concentrated closer to the midplane, with a scale height that is half of that of the atmospheric grains.

$$
H_{\text {mid }}(r)=\frac{1}{2} H_{a t m}(r)
$$

With the dust density described above, the radiative transfer code RADMC-3D (Dullemond 2012) was then used to compute the dust temperature throughout the disk. The dust absorption and scattering opacities, which are needed to solve the thermal balance, were computed using the OpacityToo ${ }^{2}$ from the DIANA project (Woitke et al. 2016). The code assumes a mixture of amorphous laboratory silicates with amorphous carbon and $25 \%$ porosity for the grain composition. The grain size distribution follows a power-law of index -3.5 . The minimum grain size was set to $5 \mathrm{~nm}$, and the maximum size was set to $10 \mu \mathrm{m}$ and $1 \mathrm{~cm}$, for the atmosphere and midplane grain populations, respectively.

The gas temperature is parametrized as

$$
T_{\text {gas }}(r, z)= \begin{cases}T_{a}+\left(T_{m}-T_{a}\right)\left(\cos \left(\frac{\pi z}{2 z_{q}}\right)\right)^{2 \delta} & z<z_{q} \\ T_{a} & z \geq z_{q}\end{cases}
$$

following Dartois et al. (2003). Here, the atmospheric temperature is given by a power-law $\left(T_{a}=\right.$ $\left.T_{a, 0}(r / 10)^{q_{a t m}}\right)$, and the midplane temperature is fixed to the dust temperature $\left(T_{m}=T_{\text {dust }}(z=0)\right)$. The fiducial scale height at which the gas temperature is allowed to vary vertically, $z_{q}$, is fixed to $z_{q}=2 H_{\text {gas }}$, where $H_{\text {gas }}=2 c_{s} / \Omega$ is the hydrostatic gas scale height evaluated at the midplane, $z=0$.

Once the gas temperature is obtained, the hydrostatic equation is solved to derive the gas density across the disk. For this we assume a vertically integrated gas-todust ratio at each radii of 100 . The adopted parameters for the model are listed in Table 5 The resulting gas density and temperature structures are shown in Figure 3. This model reproduces the main features of the ${ }^{12} \mathrm{CO},{ }^{13} \mathrm{CO}$ and $\mathrm{C}^{18} \mathrm{O}$ emission (see Fig. 4) well enough for the purpose of this study, assuming standard isotopic ratios. In this model, the $\mathrm{CO}$ abundance is kept constant throughout the disk, except in the cold midplane

Table 5: Adopted parameters in disk model.

\begin{tabular}{lll}
\hline Scale height & $H_{10}$ & $0.4 \mathrm{AU}$ \\
& $h$ & 1.25 \\
\hline \multirow{3}{*}{ Dust surface density } & $\Sigma_{c}$ & $0.226 \mathrm{~g} \mathrm{~cm}^{-2}$ \\
& $r_{c}$ & 1 \\
& $\Sigma_{c a v}$ & $10^{-4}$ \\
& $r_{c a v}$ & $3 \mathrm{AU}$ \\
\hline \multirow{3}{*}{ Gas temperature } & $T_{10}$ & $200 \mathrm{~K}$ \\
& $q_{a t m}$ & 0.8 \\
& $\delta$ & 2 \\
\hline
\end{tabular}

2 https://dianaproject.wp.st-andrews.ac.uk/data-resultsdownloads/fortran-package/ 

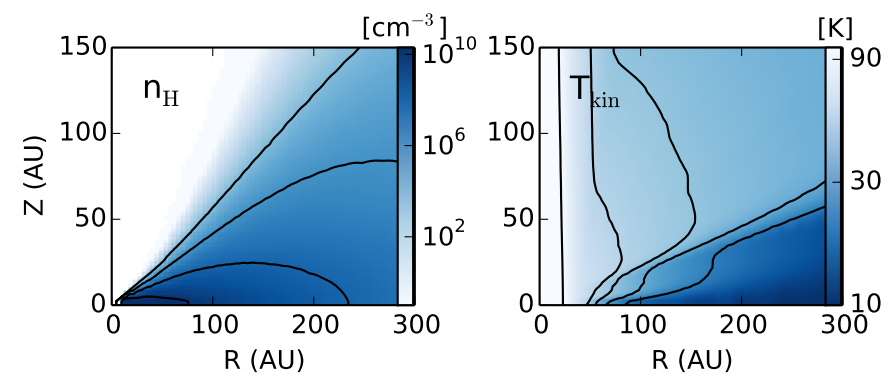

Figure 3. : Disk structure for V4046 Sgr. The contours mark densities of $10^{4}, 10^{6}, 10^{8}$ and $10^{10} \mathrm{~cm}^{-3}$ (left panel) and gas temperatures of 20, 30, 40, 50, $100 \mathrm{~K}$ (right panel).

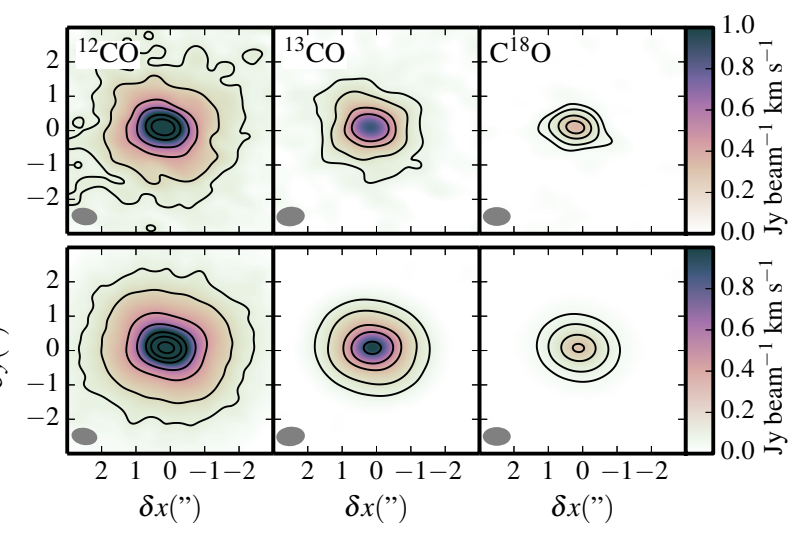

Figure 4. : Moment zero maps of the $\mathrm{CO},{ }^{13} \mathrm{CO}$ and $\mathrm{C}^{18} \mathrm{O}$ $J=2-1$ lines in V4046 Sgr. The observed and modeled integrated emission maps are shown in the upper and lower panels, respectively. The contours levels correspond to $5,10,20,30,50$ and $100 \sigma$, where the rms is 22, 18 and $10 \mathrm{mJy}$ beam $^{-1} \mathrm{kms}^{-1}$ for $\mathrm{CO},{ }^{13} \mathrm{CO}$ and $\mathrm{C}^{18} \mathrm{O}$, respectively.

$\left(T_{\text {gas }}<19 \mathrm{~K}\right)$ where the abundance is reduced by a factor of $10^{3}$ due to freeze-out onto dust grains, and in the disk atmosphere where the $\mathrm{CO}$ abundance is reduced by a factor $10^{8}$ due to photodissociation.

\subsection{Abundance fitting}

The molecular abundances for $\mathrm{H}^{13} \mathrm{CN}$ and $\mathrm{HC}^{15} \mathrm{~N}$ were defined as power-laws,

$$
X=X_{0}\left(r / R_{0}\right)^{\alpha}
$$

where $X_{0}$ is the abundance with respect to total hydrogen at $R_{0}=100 \mathrm{AU}$ and $\alpha$ is a power-law index. We also include an outer cut-off radius $R_{\text {out }}$. This parametrization is a common approach when modeling molecular abundances in disks (e.g. Qi et al. 2008, 2013).

In order to find the model that best reproduces the $\mathrm{HCN}$ isotopologue observations and the associated uncertainties, we use a Bayesian approach. In short, we first create a synthetic observation of the line emission for each species separately. Taking advantage of the bright HCN isotopologue emission in V4046 Sgr, we produced observed visibilities and cleaned cubes at a higher spectral resolution of $0.2 \mathrm{~km} \mathrm{~s}^{-1}$ for the line modeling and include include 60 channels. We use the vis_sample Python packag $\AA^{3}$ to compute the Fourier Transform of the synthetic model and obtain visibilities that are correctly reprojected on the $u-v$ points of the observations. The likelihood function is then computed in the $u-v$ plane, by computing the weighted difference between model and observations, for the real and imaginary parts of the complex visibility. We sample the posterior distribution with the MCMC method implemented in the emcee package by Foreman-Mackey et al. (2013).

We include two free parameters in the line modeling, that is $X_{0}, \alpha$, which are associated with the molecular abundances of $\mathrm{H}^{13} \mathrm{CN}$ and $\mathrm{HC}^{15} \mathrm{~N}$. The outer radius, $R_{\text {out }}$, was fixed to $100 \mathrm{AU}$ (chosen by the extension of the emission in the moment-zero map), but we checked that a larger radius of 200 AU gave the same result. The disk physical structure, that is the gas density and temperature, are fixed in the line fitting. We adopt the disk inclination, position angle, stellar mass (including both stars) and systemic velocity listed in Table 2. The level populations were computed using RADMC and assuming the gas is under LTE. We checked that non-LTE effects are not important for these lines using the non-LTE radiative transfer code LIME (Brinch \& Hogerheijde 2010) to re-calculate the level populations for the best-fit model. When generating a new sample, we included a flat prior for the power-law index $-3<\alpha<2$, and for the molecular abundance $10^{-20}<X_{100}<10^{-8}$.

The best-fit model corresponds to $X_{0}=8.94 \pm 0.30 \times$ $10^{-13}$ and $\alpha=-0.69 \pm 0.03$ for $\mathrm{H}^{13} \mathrm{CN}$, and $X_{0}=$ $3.37 \pm 0.19 \times 10^{-13}$ and $\alpha=-1.08 \pm 0.04$ for $\mathrm{HC}^{15} \mathrm{~N}$. Our model suggests an increasing $\mathrm{H}^{13} \mathrm{CN} / \mathrm{HC}^{15} \mathrm{~N}$ ratio as a function of radius, i.e. higher fractionation in the inner disk compared to the outer disk. Fig. 5 shows the deprojected radial profiles of the dust continuum and $\mathrm{HCN}$ isotopologue emission in V4046 Sgr (left panel) as well as the observed and modeled $\mathrm{H}^{13} \mathrm{CN} / \mathrm{HC}^{15} \mathrm{~N}$ flux ratio (right panel). We note that beyond $60 \mathrm{AU}$, the ratio is highly uncertain because the signal-to-noise ratio becomes too low, in particular for $\mathrm{HC}^{15} \mathrm{~N}$. Figure 6 shows
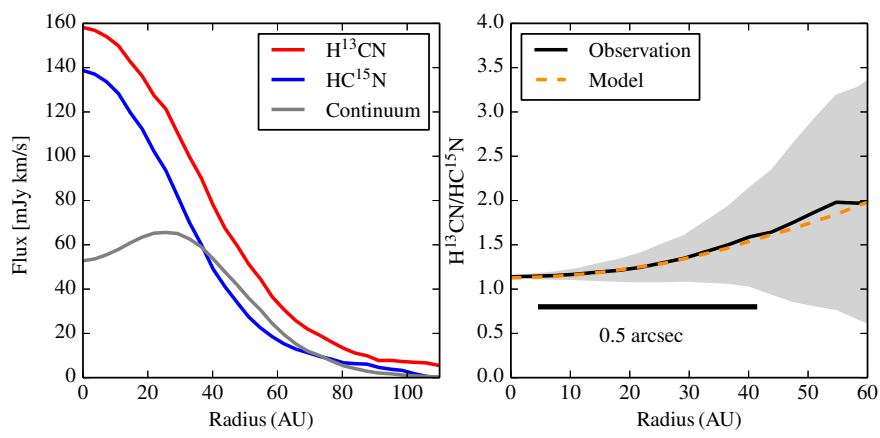

Figure 5. : Left: Azimuthally-averaged emission profiles of the dust continuum emission (gray), $\mathrm{H}^{13} \mathrm{CN} J=3-2$ (red) and $\mathrm{HC}^{15} \mathrm{~N} J=3-2$ (blue) lines in V4046 Sgr. Right: The observed $\mathrm{H}^{13} \mathrm{CN} / \mathrm{HC}^{15} \mathrm{~N}$ flux ratio (black) and the ratio derived from our best-fit model (dashed orange). The error in the observed ratio (gray ribbon) was computed by taking the standard deviation of the $\mathrm{H}^{13} \mathrm{CN}$ and $\mathrm{HC}^{15} \mathrm{~N}$ fluxes at each ratio. The black horizontal bar shows the beam size of $0^{\prime \prime} .5$.

3 https://pypi.python.org/pypi/vis_sample 

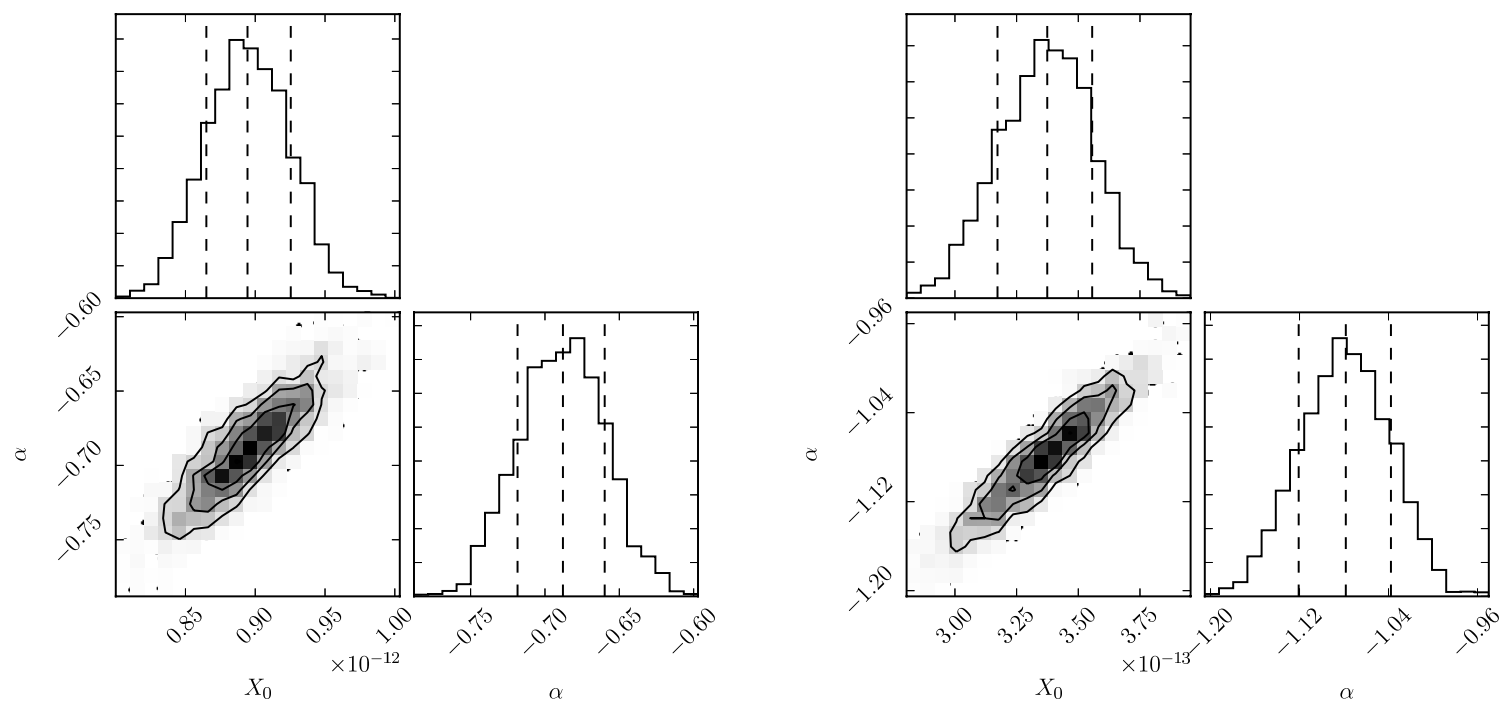

Figure 6. : Line modeling results of $\mathrm{H}^{13} \mathrm{CN}$ (left) are $\mathrm{HC}^{15} \mathrm{~N}$ (right) for the disk around V4046 Sgr. The gray color scales show the joint probability distribution probabilities for the parameters, $\alpha$ and $X_{0}$. The contours represent the $1 \sigma, 2 \sigma$ and $3 \sigma$ levels. The histograms show the marginal distribution for each parameter and the vertical dashed lines mark the median and $1 \sigma$ uncertainty.
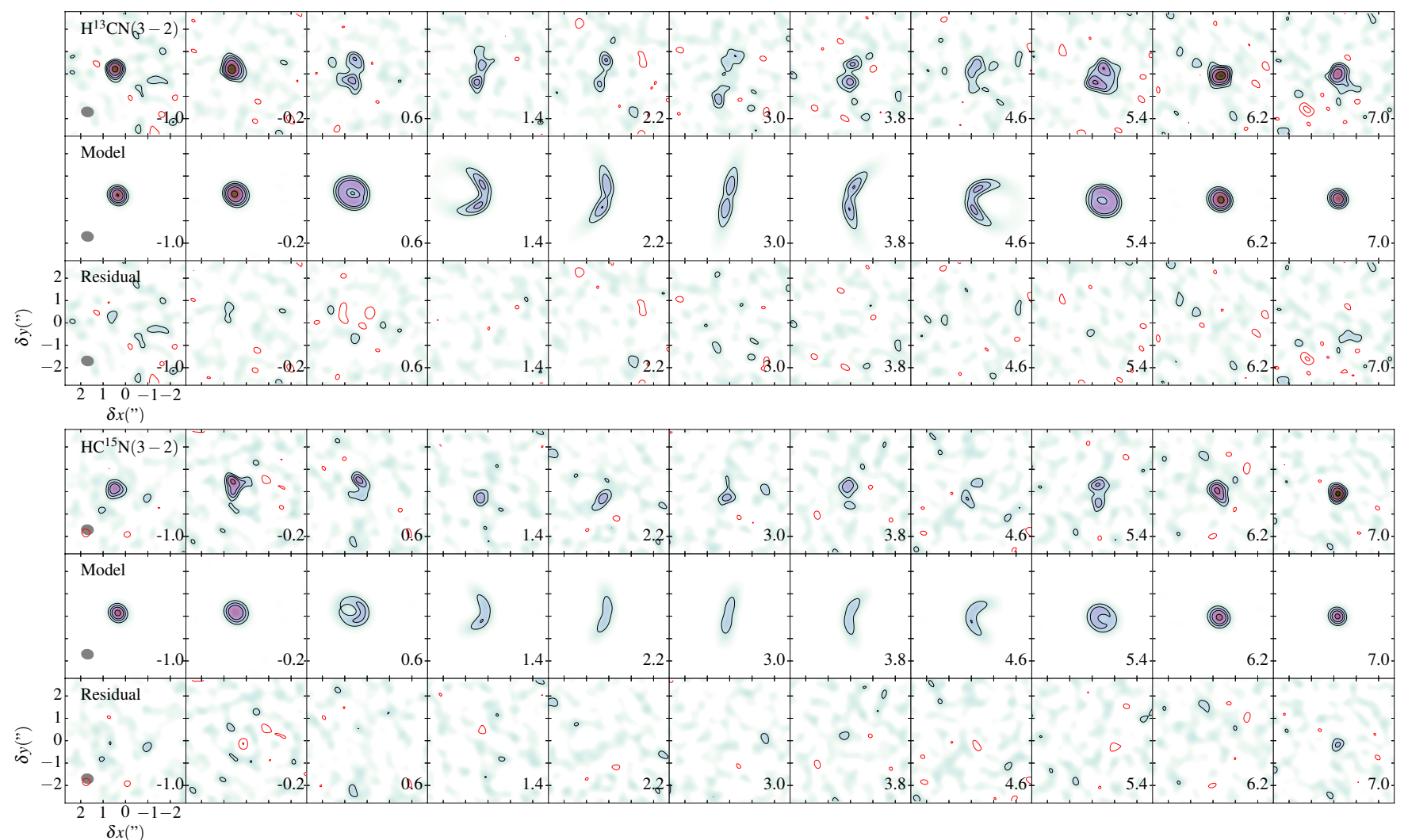

Figure 7. : Channel maps of the observed $\mathrm{H}^{13} \mathrm{CN}$ and $\mathrm{HC}^{15} \mathrm{~N} J=3-2$ emission lines in V4046 Sgr (upper panels). The middle panels show the best-fit models for each line. The residuals are shown in the bottom panels. Positive (black) and negative (red) contour levels correspond to 3, 5, 7 10, 15, 20 and $25 \sigma$. 
the posterior probability distribution for the fitted parameters. Fig. 7 shows the residuals between our best-fit model and the data for selected channels. Our simple model is able to well reproduce the observations.

The inferred abundance $\mathrm{H}^{13} \mathrm{CN} / \mathrm{HC}^{15} \mathrm{~N}$ ratio at 10 and $50 \mathrm{AU}$ are $1.08 \pm 0.14$ and $2.02 \pm 0.27$, respectively. Assuming ${ }^{12} \mathrm{C} /{ }^{13} \mathrm{C}=70 \pm 21$, we infer an abundance ${ }^{14} \mathrm{~N} /{ }^{15} \mathrm{~N}$ ratio of $76 \pm 25$ and $142 \pm 47$, at 10 and $50 \mathrm{AU}$ respectively. We note that the derived disk integrated $\mathrm{HCN} / \mathrm{HC}^{15} \mathrm{~N}$ flux ratio of $115 \pm 43$ falls in between the inferred abundance ratios at 10 and $50 \mathrm{AU}$. Given the consistent nitrogen fractionation levels inferred from the observations and line modeling in V4046 Sgr, we expect the observed ${ }^{14} \mathrm{~N} /{ }^{15} \mathrm{~N}$ ratio in the other sources to be representative of their abundance ratio in the comet forming region.

\section{DISCUSSION}

\subsection{Disk-averaged nitrogen fractionation in protoplanetary disks}

We have shown that $\mathrm{HCN}$ isotopologues are abundant in disks. Both $\mathrm{H}^{13} \mathrm{CN}$ and $\mathrm{HC}^{15} \mathrm{~N}$ are detected toward $5 / 6$ disks in our sample - the one exception being the disk around T Tauri star IM Lup. The disk around IM Lup is very massive $\left(\mathrm{M}_{\text {disk }}=0.1 \mathrm{M}_{\odot}\right)$, very cold and also very young (e.g., Cleeves et al. 2016) compared to the rest of the disks in the sample. The non-detection of $\mathrm{H}^{13} \mathrm{CN}$ was surprising considering that IM Lup is quite bright in the main HCN isotopologue (Öberg et al. 2011). Given the observed HCN flux density of $3.5 \mathrm{Jy} \mathrm{km} \mathrm{s}^{-1}$, we could expect a $\mathrm{H}^{13} \mathrm{CN}$ flux density of $50 \mathrm{mJy} \mathrm{km} \mathrm{s}^{-1}$ if $\mathrm{HCN}$ is optically thin and ${ }^{12} \mathrm{C} /{ }^{13} \mathrm{C}=70$. This is consistent with the observed $3 \sigma$ upper limit of $51 \mathrm{mJy} \mathrm{km} \mathrm{s}^{-1}$.

The inferred disk-averaged nitrogen fractionation ratios range from $83 \pm 37$ to $156 \pm 78$. Despite the different physical conditions of the disks in the sample, the observed $\mathrm{H}^{13} \mathrm{CN} / \mathrm{HC}^{15} \mathrm{~N}$ ratios are consistent with sampling a constant disk-averaged fractionation level. In particular, we find no difference in the nitrogen fractionation level between disks around T Tauri and Herbig Ae stars, which have an order of magnitude difference in the stellar radiation field. No difference in the disk-averaged ${ }^{14} \mathrm{~N} /{ }^{15} \mathrm{~N}$ is observed between full and transitional disks, either. The age of the star does not seem to play an important role either - we target young $(\sim 1 \mathrm{Myr})$ and old $\left(>10 \mathrm{Myr}\right.$ ) sources - suggesting either that the ${ }^{14} \mathrm{~N} /{ }^{15} \mathrm{~N}$ is inherited from the parent cloud and is not modified in the disk, or the disk chemistry sets the global, disk averaged nitrogen fractionation level early $(\lesssim 1 \mathrm{Myr})$ in the protoplanetary disk life.

Although we do not observe differences in the nitrogen fractionation ratio between the sources, the data suggest that there may be a difference in the nitrogen abundance between the disks around stars V4046 Sgr, MWC 480 and HD 163206, and the disks around T Tauri stars AS 209 and $\mathrm{LkCa} 15$. The old disk around the binary $\mathrm{T}$ Tauri stars V4046 Sgr and the two Herbig Ae stars in the sample are enriched in HCN compared to the young T Tauri stars. Disk models have shown that dust migration and carbon and oxygen depletion (mainly due to $\mathrm{CO}$ and $\mathrm{H}_{2} \mathrm{O}$ freeze-out) can increase the column density of cyanides by up to two orders of magnitude in the outer disk (Du et al. 2015). Future observations toward a larger sample of disks will show if this corresponds to an evolutionary trend.

\subsection{Comparison between nitrogen fractionation in disks, Solar System and ISM}

Fig. 8 shows the observed ${ }^{14} \mathrm{~N} /{ }^{15} \mathrm{~N}$ ratios in different Solar System bodies, the cold ISM and in the diffuse medium. There are large variations in the ${ }^{14} \mathrm{~N} /{ }^{15} \mathrm{~N}$ ratio among different Solar system bodies, in particular between the rocky and gaseous bodies. The Sun has the highest ${ }^{14} \mathrm{~N} /{ }^{15} \mathrm{~N}$ value in this comparison $(441 \pm 5)$, measured by the Genesis mission that sampled solar wind ions, $\mathrm{N}^{+}$among them (Marty et al. 2011). An almost identical value was found in the atmosphere of Jupiter through the $\mathrm{NH}_{3}$ observations carried-out by the Cassini spacecraft (Fouchet et al. 2004). Both measurements are expected to trace the lack of nitrogen fractionation in $\mathrm{N}_{2}$, the main nitrogen reservoir of the protosolar nebula. The ${ }^{15} \mathrm{~N}$-depleted Solar value is thus considered to be representative of the conditions of the gas when the Sun formed. All the other Solar system bodies are enriched in ${ }^{15} \mathrm{~N}$ compared to the Sun. A value of ${ }^{14} \mathrm{~N} /{ }^{15} \mathrm{~N}=272$ is found in the Earth's atmosphere, measured in $\mathrm{N}_{2}$. The ${ }^{14} \mathrm{~N} /{ }^{15} \mathrm{~N}$ has also been measured in several comets. An isotopic ratio of $\sim 150$ was found in C/1995 O1 (Hale-Bopp) and 17P/Holmes, value which was consistent for both $\mathrm{HCN}$ and $\mathrm{CN}$ (Bockelée-Morvan et al. 2008). Observations of 18 comets from both the Oort cloud and the Kuiper Belt all show consistently low $\mathrm{HCN} / \mathrm{HC}^{15} \mathrm{~N} \simeq 100-250$ ratios (Mumma \& Charnley 2011).

The cold interstellar medium is also enriched in ${ }^{15} \mathrm{~N}$. Hily-Blant et al. (2013) measured the $\mathrm{H}^{13} \mathrm{CN} / \mathrm{HC}^{15} \mathrm{~N}$ toward two prestellar cores, L183 and L1544, and found ${ }^{14} \mathrm{~N} /{ }^{15} \mathrm{~N}$ values of $140-250$ and $140-360$, respectively. The ${ }^{14} \mathrm{~N} /{ }^{15} \mathrm{~N}$ in $\mathrm{CN}$ was later measured toward L1544 resulting in a surprisingly high $\mathrm{CN} / \mathrm{C}^{15} \mathrm{~N}$ ratio of $500 \pm 75$ (Hily-Blant et al. 2013b). The authors were able to reproduce the observed difference in $\mathrm{CN}$ and $\mathrm{HCN}$ with chemical models of cold gas. The fact that $\mathrm{CN}$ and $\mathrm{HCN}$

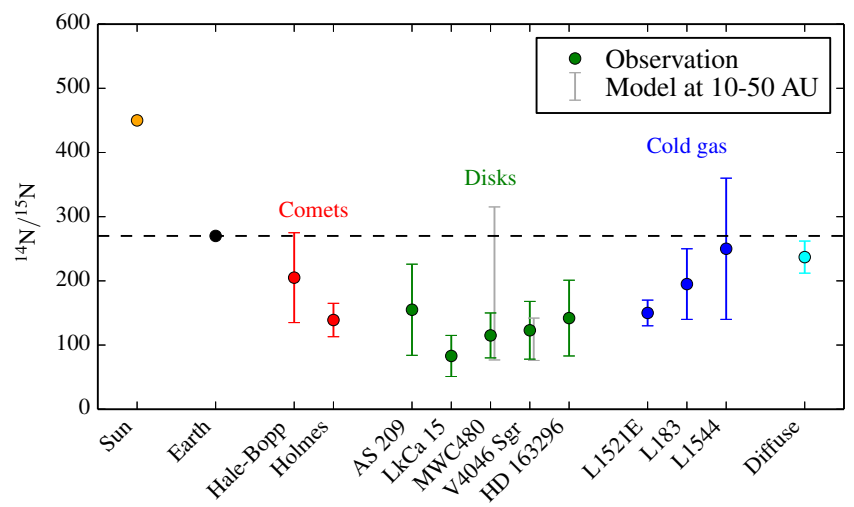

Figure 8. : ${ }^{14} \mathrm{~N} /{ }^{15} \mathrm{~N}$ isotopic ratios observed toward different solar system bodies (Marty et al. 2011; BockeléeMorvan et al. 2008), the ISM (Hily-Blant et al. 2013; Ikeda et al. 2002; Lucas \& Liszt 1998), and protoplanetary disks (this work). The Solar and Earth values were measured for $\mathrm{N}^{+}$and $\mathrm{N}_{2}$, respectively, the rest of the sources all correspond to isotopic ratios measured for $\mathrm{HCN}$. 
present similar fractionation levels in comets, could be explained if $\mathrm{CN}$ is produced in the coma from photodissociation of HCN (Hily-Blant et al. 2013b). Ikeda et al. (2002) also found a low $\mathrm{HCN} / \mathrm{HC}^{15} \mathrm{~N}$ ratio of $151 \pm 16$ toward the prestellar core L1521E.

Finally, the averaged ${ }^{15} \mathrm{~N}$ enrichment observed for $\mathrm{HCN}$ in protoplanetary disks are similar to comets and the cold ISM. While the similar nitrogen fractionation ratios found in the cold ISM, comets and disks is consistent with an inheritance scenario for the origin of organics in the Solar System, as we discuss in the next section, the increasing ${ }^{14} \mathrm{~N} /{ }^{15} \mathrm{~N}$ ratio in the disk of V4046 Sgr suggests that in situ disk chemistry also contributes to the observed fractionation patterns.

\subsection{Resolved nitrogen fractionation chemistry in disks}

The bright emission of the $\mathrm{HCN}$ isotopologues in V4046 Sgr allows us to trace the ${ }^{14} \mathrm{~N} /{ }^{15} \mathrm{~N}$ profile across a disk for the first time. In general, there are three possibilities for what could be observed: a flat, decreasing or increasing ${ }^{14} \mathrm{~N} /{ }^{15} \mathrm{~N}$ as a function of disk radius. If $\mathrm{H}^{13} \mathrm{CN}$ and $\mathrm{HC}^{15} \mathrm{~N}$ are inherited from the prestellar gas and no further chemical processing occurs in the disk, then a constant ${ }^{14} \mathrm{~N} /{ }^{15} \mathrm{~N}$ ratio is expected across the disk. On the other hand, if the nitrogen chemistry is altered by in-situ chemical fractionation in the disk then a varying ${ }^{14} \mathrm{~N} /{ }^{15} \mathrm{~N}$ is expected. In this case, if chemical fractionation dominates the nitrogen fractionation then a low ${ }^{14} \mathrm{~N} /{ }^{15} \mathrm{~N}$ is expected to occur in the outer disk, where the gas temperature is low (i.e., a decreasing ${ }^{14} \mathrm{~N} /{ }^{15} \mathrm{~N}$ with radius). In contrast, if selective photodissociation is the dominant pathway to fractionate $\mathrm{HCN}$, then an increasing ${ }^{14} \mathrm{~N} /{ }^{15} \mathrm{~N}$ profile would be observed because this pathway is most important in regions exposed to UV photons, i.e. the inner disk which is illuminated by the central star.

The observations toward V4046 Sgr show that both $\mathrm{H}^{13} \mathrm{CN}$ and $\mathrm{HC}^{15} \mathrm{~N}$ are best reproduced by a decreasing abundance profile. However, the inferred $\mathrm{HC}^{15} \mathrm{~N}$ emission profile is slightly steeper than that of $\mathrm{H}^{13} \mathrm{CN}$, pointing to a higher fractionation in the inner disk than in the outer disk. The varying ${ }^{14} \mathrm{~N} /{ }^{15} \mathrm{~N}$ observed in V4046 Sgr shows that there is an active nitrogen fractionation in the disk, that changes the original fractionation pattern. The fact that ${ }^{14} \mathrm{~N} /{ }^{15} \mathrm{~N}$ is lower in the inner disk suggests that selective photodissociation is indeed an important pathway to fractionate $\mathrm{HCN}$ in the inner disk. Higher signal-to-noise ratio observations are needed to determine if there is also an active $\mathrm{N}$ fractionation chemistry in the outer disk.

There is additional evidence that cold ion-molecule fractionation does not alone regulate the $\mathrm{H}^{13} \mathrm{CN} / \mathrm{HC}^{15} \mathrm{~N}$ ratio in disks. Huang et al. (2017) measured the $\mathrm{D} / \mathrm{H}$ isotopic ratio in $\mathrm{DCO}^{+}$and $\mathrm{HCN}$ toward the same sample of disks. They found enhanced $\mathrm{D} / \mathrm{H}$ ratios compared to the elemental ratio in the local ISM $\left(\sim 2 \times 10^{-5}\right)$ in all disks, with DCN/HCN ratios ranging from 0.005-0.08. If the cold pathway dominates the fractionation for both species in these disks we could expect a correlation between the $\mathrm{D} / \mathrm{H}$ and ${ }^{14} \mathrm{~N} /{ }^{15} \mathrm{~N}$ ratios. However, we do not see such correlation in the sample (see Fig. 2).

\subsection{Future directions}

We have shown that the ${ }^{14} \mathrm{~N} /{ }^{15} \mathrm{~N}$ ratio increases with radius in the disk around T Tauri stars V4046 Sgr. However, the angular resolution of the current data $\left(0^{\prime \prime} .5\right.$ corresponding to $\sim 36 \mathrm{AU}$ at $73 \mathrm{pc}$ ) prevents us from resolving ${ }^{14} \mathrm{~N} /{ }^{15} \mathrm{~N}$ variations at smaller scales and the low signal-to-noise ratio prevents us from constraining the chemistry beyond 60 AU. Future observations at higher angular resolution should allow us to measure the radial dependence of ${ }^{14} \mathrm{~N} /{ }^{15} \mathrm{~N}$ at Solar System scales, and determine how the fractionation ratio changes from the inner $(<15 \mathrm{AU})$ to the outer $(>30 \mathrm{AU})$ disk.

High-angular resolution observations toward more disks are needed to determine whether an increasing ${ }^{14} \mathrm{~N} /{ }^{15} \mathrm{~N}$ is a general characteristic of disks or unique to V4046 Sgr. A larger sample is also needed to draw more general conclusions on the dominant fractionation pathways in disks, and how the ${ }^{14} \mathrm{~N} /{ }^{15} \mathrm{~N}$ pattern depends on the physical conditions of the disk. In this respect, new chemical models that include all nitrogen fractionation pathways (selective photodissociation and cold isotope exchange reactions) as well as inheritance from the parent cloud are key to interpret the observations. In addition, it is desirable to include the fractionation of both carbon and nitrogen in the models, since most ${ }^{14} \mathrm{~N} /{ }^{15} \mathrm{~N}$ measurements in both disks and cold ISM rely on observations of the ${ }^{13} \mathrm{C}$ isotopologues to measure the contribution of the main isotopologues.

In contrast to $\mathrm{HCN}$, hydrides (e.g., $\mathrm{N}_{2} \mathrm{H}^{+}$and $\mathrm{NH}_{3}$ ) are found to be ${ }^{15} \mathrm{~N}$-depleted in the ISM. Toward L1544, Bizzocchi et al. (2013) found a nitrogen isotopic ratio in $\mathrm{N}_{2} \mathrm{H}^{+}$of 1000 . Toward the class 0 protostar B1b, Daniel et al. (2013) found ${ }^{14} \mathrm{~N} /{ }^{15} \mathrm{~N}$ ratios of $260-355$ for $\mathrm{NH}_{3}$ and an upper limit of $>600$ for $\mathrm{N}_{2} \mathrm{H}^{+}$. HilyBlant et al. (2013) proposed that the difference in nitrogen fractionation between cyanides and hydrides in the cold ISM is the result of their different chemical origins: $\mathrm{HCN}$ derives from atomic $\mathrm{N}$, while $\mathrm{NH}_{3}$ and $\mathrm{N}_{2} \mathrm{H}^{+}$derive from molecular nitrogen. This hypothesis, however, is challenged by the recent measurement of the ${ }^{14} \mathrm{~N} /{ }^{15} \mathrm{~N}$ in $\mathrm{NH}_{2}$, a photodissociation product of $\mathrm{NH}_{3}$ in cometary coma, toward comet C/2012 S1 (ISON), where a fractionation ratio of $139 \pm 38$ was found (Shinnaka et al. 2014). A similarly low value $(\sim 130)$ was found previously by Rousselot et al. (2014) based on the averaged spectrum of 12 comets. Toward comet ISON, CN and HCN were also found to be highly enriched in ${ }^{15} \mathrm{~N}\left({ }^{14} \mathrm{~N} /{ }^{15} \mathrm{~N} \sim 150\right)$. As explained by Shinnaka et al. (2014), one possibility to obtain similar $\mathrm{HCN}$ and $\mathrm{NH}_{3}$ fractionation levels in comets, despite very different fractionation levels in the cold ISM, is through grain surface chemistry. Indeed, the ISM values represent the ${ }^{14} \mathrm{~N} /{ }^{15} \mathrm{~N}$ in the gas-phase, while cometary values represent the ${ }^{14} \mathrm{~N} /{ }^{15} \mathrm{~N}$ in the ices. It is also possible that the measured cometary values is not representative of the composition of the nucleus. Measurements of the isotopic ratio in $\mathrm{NH}_{3}$ and $\mathrm{N}_{2} \mathrm{H}^{+}$in disks would provide additional clues to answer these questions. In particular, if the cyanide/hydride dichotomy observed in the cold ISM holds for disks as well.

Finally, more observations toward comets targeting different molecular parent species (i.e., species tracing the cometary nucleus and not daughter species which are produced in the coma) will be important to compare with observations in the ISM and disks, and to elucidate the 
origins of ${ }^{15} \mathrm{~N}$ enhancements, and ultimately the origins of organics, across the Solar System.

\section{CONCLUSIONS}

We have presented ALMA observations at $\sim 0^{\prime \prime} .5$ angular resolution of the $\mathrm{HCN}$ isotopologues in a diverse sample of six protoplanetary disks. The sample contains $4 \mathrm{~T}$ Tauri and 2 Herbig Ae stars, which sample an order of magnitude in radiation fields. Both $\mathrm{H}^{13} \mathrm{CN}$ and $\mathrm{HC}^{15} \mathrm{~N}$ are detected toward all the sources, except for IM Lup, which is the most massive and coldest disk, and likely the youngest, in the sample. Adopting a standard ${ }^{12} \mathrm{C} /{ }^{13} \mathrm{C}$ ratio of 70 (with a $30 \%$ uncertainty), we infer disk-averaged ${ }^{14} \mathrm{~N} /{ }^{15} \mathrm{~N}$ ratios of $80-160$ for the sources. Despite the different physical conditions of the disks, ${ }^{14} \mathrm{~N} /{ }^{15} \mathrm{~N}$ in $\mathrm{HCN}$ is similar for all sources. No differences are observed between $\mathrm{T}$ Tauri and Herbig Ae stars, or between the full disks and the transitional disks, which feature large dust cavities. Also, no correlation is observed between disk-averaged $\mathrm{D} / \mathrm{H}$ and ${ }^{14} \mathrm{~N} /{ }^{15} \mathrm{~N}$ ratios in the sample. The observed disk-averaged ${ }^{14} \mathrm{~N} /{ }^{15} \mathrm{~N}$ ratios are similar to what is observed in comets and in the cold ISM, which is consistent with the inheritance scenario for the origin of organics in the Solar System. However, chemical processing within the protoplanetary disk phase based on these ratios alone cannot be ruled out. Indeed, in the one disk where we could resolve the $\mathrm{H}^{13} \mathrm{CN} / \mathrm{HC}^{15} \mathrm{~N}$ ratio as a function of radius we find a slightly steeper profile for $\mathrm{HC}^{15} \mathrm{~N}$, i.e. an increasing ${ }^{14} \mathrm{~N} /{ }^{15} \mathrm{~N}$ ratio with radius. The higher nitrogen fractionation level in the inner disk compared to the outer disk suggest that selective photodissociation is an important fractionation pathway in the inner disk.

This paper makes use of ALMA data, project code: ADS/JAO.ALMA\#2013.1.00226. ALMA is a partnership of ESO (representing its member states), NSF (USA) and NINS (Japan), together with NRC (Canada) and NSC and ASIAA (Taiwan), in cooperation with the Republic of Chile. The Joint ALMA Observatory is operated by ESO, AUI/NRAO and NAOJ. The National Radio Astronomy Observatory is a facility of the National Science Foundation operated under cooperative agreement by Associated Universities, Inc. VVG thanks support from the Chilean Government through the Becas Chile program. KIÖ also acknowledges funding from the Packard Foundation and an investigator award from Simons Collaboration on the Origins of Life (SCOL). JH and RL acknowledge support from the National Science Foundation (Grant No. DGE-1144152).
Du, F., Bergin, E. A., \& Hogerheijde, M. R. 2015, ApJ, 807, L32

Bizzocchi, L., Caselli, P., Leonardo, E., \& Dore, L. 2013, A\&A, 555, A109

Bockelée-Morvan, D., Biver, N., Jehin, E., et al. 2008, ApJ, 679, L49

Brinch, C., \& Hogerheijde, M. R. 2010, A\&A, 523, A25

Chapillon, E., Guilloteau, S., Dutrey, A., Piétu, V., \& Guélin, M. 2012, A\&A, 537, A60

Cleeves, L. I., Öberg, K. I., Wilner, D. J., et al. 2016, arXiv:1610.00715

Daniel, F., Gérin, M., Roueff, E., et al. 2013, A\&A, 560, A3

Dartois, E., Dutrey, A., \& Guilloteau, S. 2003, A\&A, 399, 773

Dullemond, C. P. 2012, Astrophysics Source Code Library, ascl: 1202.015

Foreman-Mackey, D., Hogg, D. W., Lang, D., \& Goodman, J. 2013, PASP, 125, 306

Fouchet, T., Irwin, P. G. J., Parrish, P., et al. 2004, Icarus, 172, 50

Guzmán, V. V., Öberg, K. I., Loomis, R., \& Qi, C. 2015, ApJ, 814,53

Heays, A. N., Visser, R., Gredel, R., et al. 2014, A\&A, 562, A61

Hily-Blant, P., Bonal, L., Faure, A., \& Quirico, E. 2013, Icarus, 223,582

Hily-Blant, P., Pineau des Forêts, G., Faure, A., Le Gal, R., \& Padovani, M. 2013, A\&A, 557, A65

Huang, J., Oberg, K. I., Qi, C., et al. 2017, arXiv:1701.01735

Ikeda, M., Hirota, T., \& Yamamoto, S. 2002, ApJ, 575, 250

Lyons, J. R., Bergin, E. A., Ciesla, F. J., et al. 2009,

Geochim. Cosmochim. Acta, 73, 4998

Lucas, R., \& Liszt, H. 1998, A\&A, 337, 246

Liszt, H., \& Lucas, R. 2001, A\&A, 370, 576

Marty, B., Chaussidon, M., Wiens, R. C., Jurewicz, A. J. G., \& Burnett, D. S. 2011, Science, 332, 1533

Mumma, M. J., \& Charnley, S. B. 2011, ARA\&A, 49, 471

Öberg, K. I., Furuya, K., Loomis, R., et al. 2015, ApJ, 810, 112

Öberg, K. I., Guzmán, V. V., Furuya, K., et al. 2015, Nature, 520,198

Öberg, K. I., Qi, C., Fogel, J. K. J., et al. 2010, ApJ, 720, 480

Öberg, K. I., Qi, C., Fogel, J. K. J., et al. 2011, ApJ, 734, 98

Pavlyuchenkov, Y., Semenov, D., Henning, T., et al. 2007, ApJ, 669, 1262

\& Guilloteau, S. 2007, A\&A, 467, 163

Rosenfeld, K. A., Andrews, S. M., Wilner, D. J., Kastner, J. H., \& McClure, M. K. 2013, ApJ, 775, 136

Roueff, E., Loison, J. C., \& Hickson, K. M. 2015, A\&A, 576, A99

Rousselot, P., Pirali, O., Jehin, E., et al. 2014, ApJ, 780, L17

Shinnaka, Y., Kawakita, H., Kobayashi, H., Nagashima, M., \& Boice, D. C. 2014, ApJ, 782, L16

Thi, W.-F., van Zadelhoff, G.-J., \& van Dishoeck, E. F. 2004, A\&A, 425, 955

Qi, C., Öberg, K. I., \& Wilner, D. J. 2013, ApJ, 765, 34

Qi, C., Wilner, D. J., Aikawa, Y., Blake, G. A., \& Hogerheijde, M. R. 2008, ApJ, 681, 1396-1407

Wampfler, S. F., Jørgensen, J. K., Bizzarro, M., \& Bisschop, S. E. 2014, A\&A, 572, A24

Woitke, P., Min, M., Pinte, C., et al. 2016, A\&A, 586, A103

\section{REFERENCES}




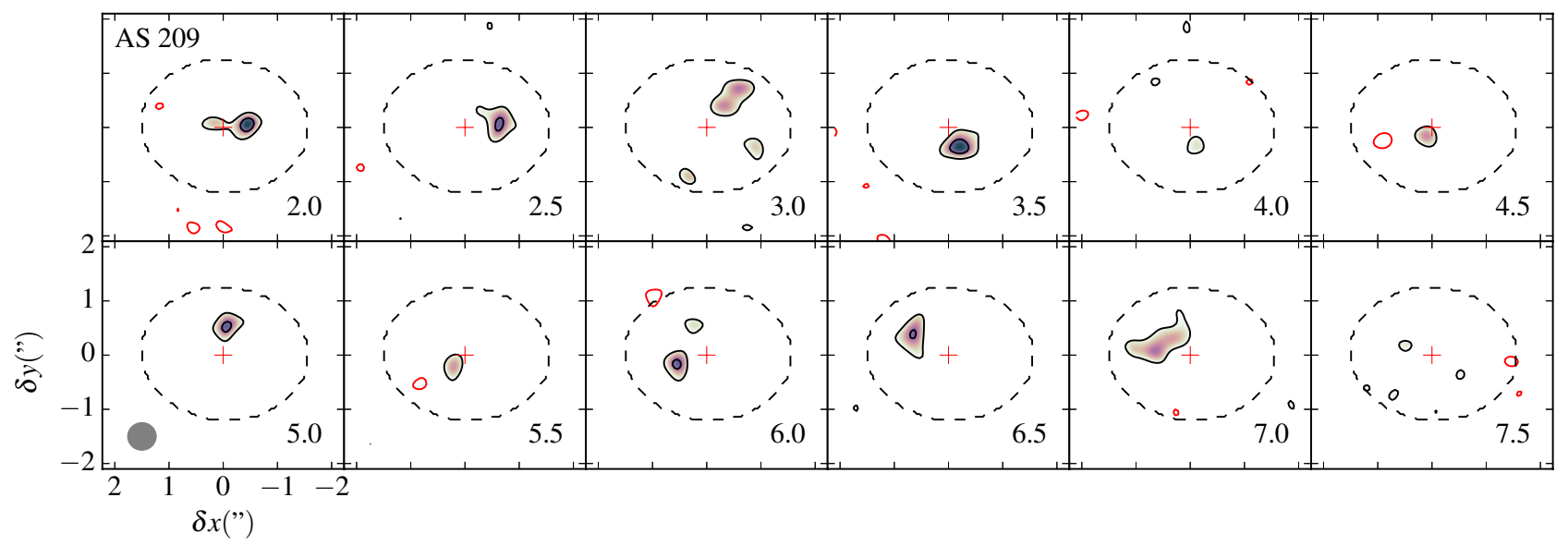

(a) $\mathrm{H}^{13} \mathrm{CN}$

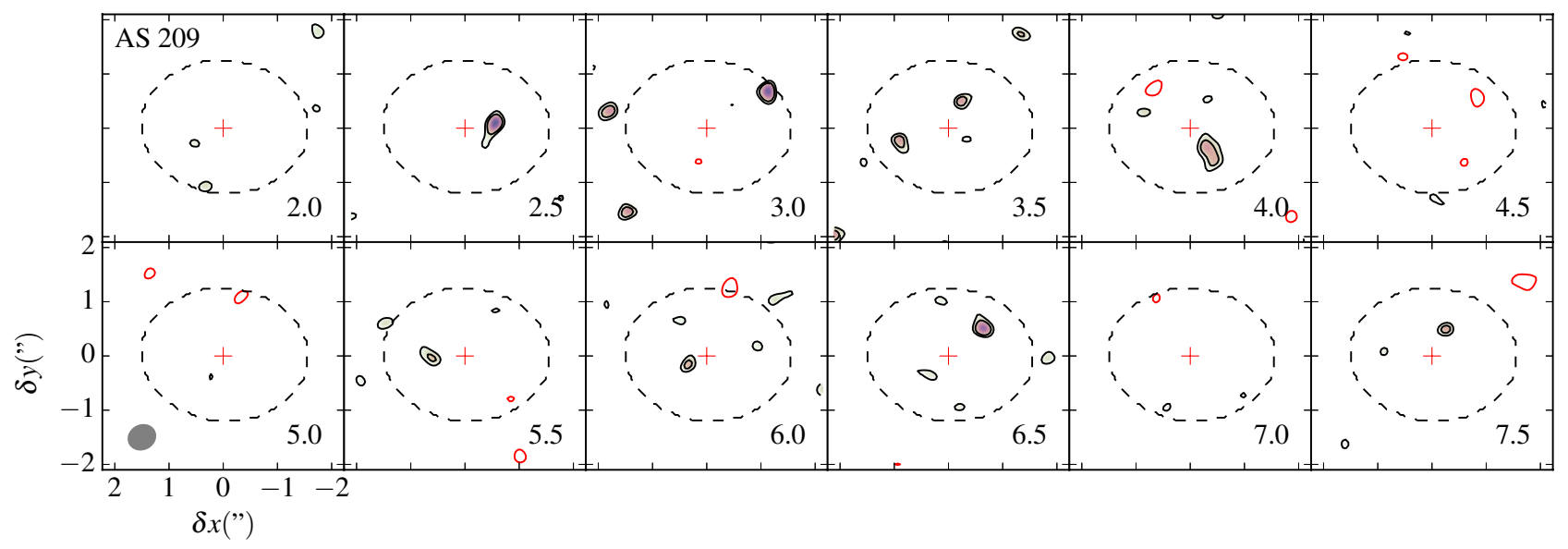

(b) $\mathrm{HC}^{15} \mathrm{~N}$

Figure A.1. : Channel maps of the observed $\mathrm{H}^{13} \mathrm{CN}$ (upper) and $\mathrm{HC}^{15} \mathrm{~N}$ (lower) $J=3-2$ emission lines in the disk around star AS 209. The contour levels correspond to 3, 5, $710,15,20$ and $25 \times$ rms, with rms given in Table 1 . Positive and negative contours are drawn in black and red, respectively. For $\mathrm{HC}^{15} \mathrm{~N}$, an additional contour corresponding to $2.5 \times \mathrm{rms}$ is drawn. The elliptical mask used to extract the spectra and integrated flux is overlaid in dashed lines. The red crosses mark the continuum image centroid. The synthesized beam is shown in the bottom left panels. 


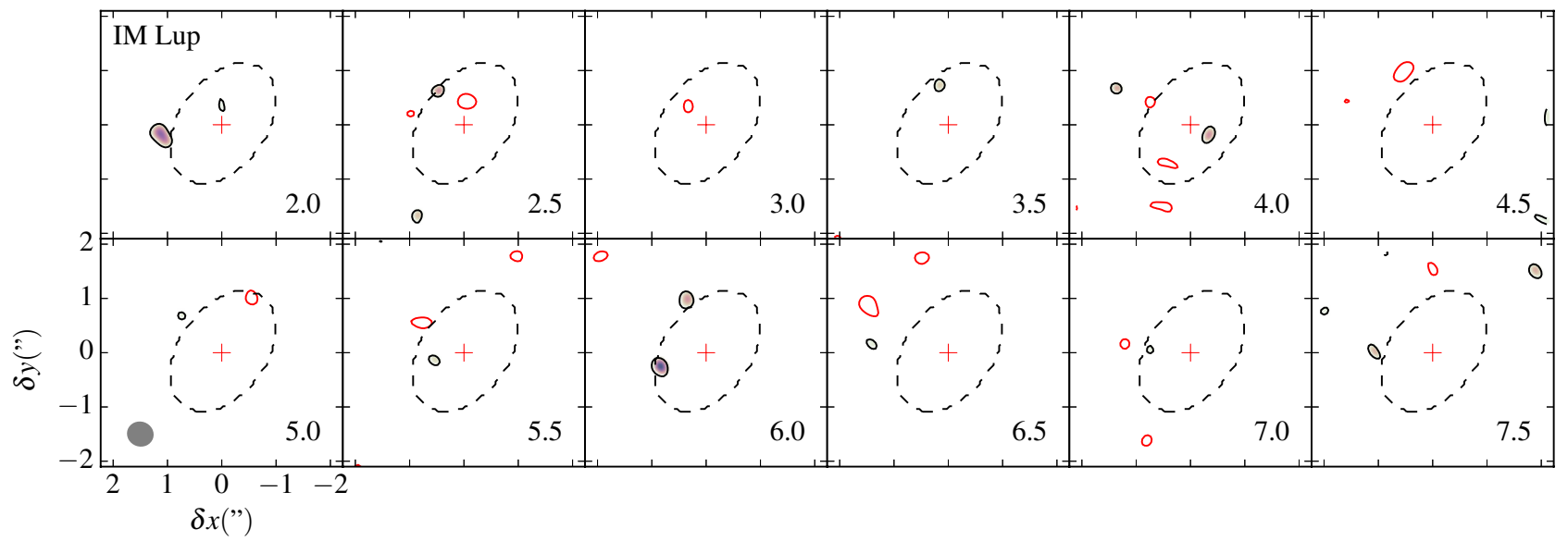

(a) $\mathrm{H}^{13} \mathrm{CN}$

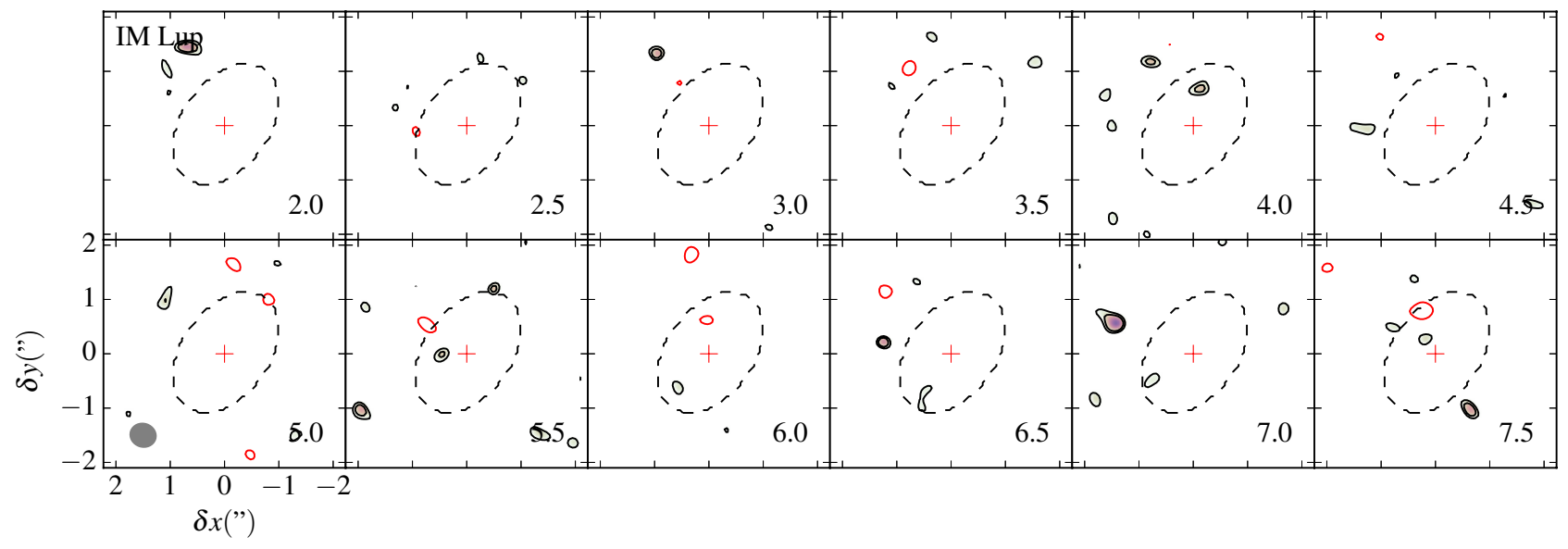

(b) $\mathrm{HC}^{15} \mathrm{~N}$

Figure A.2. : Same as in Fig. A.1 but for IM Lup. 


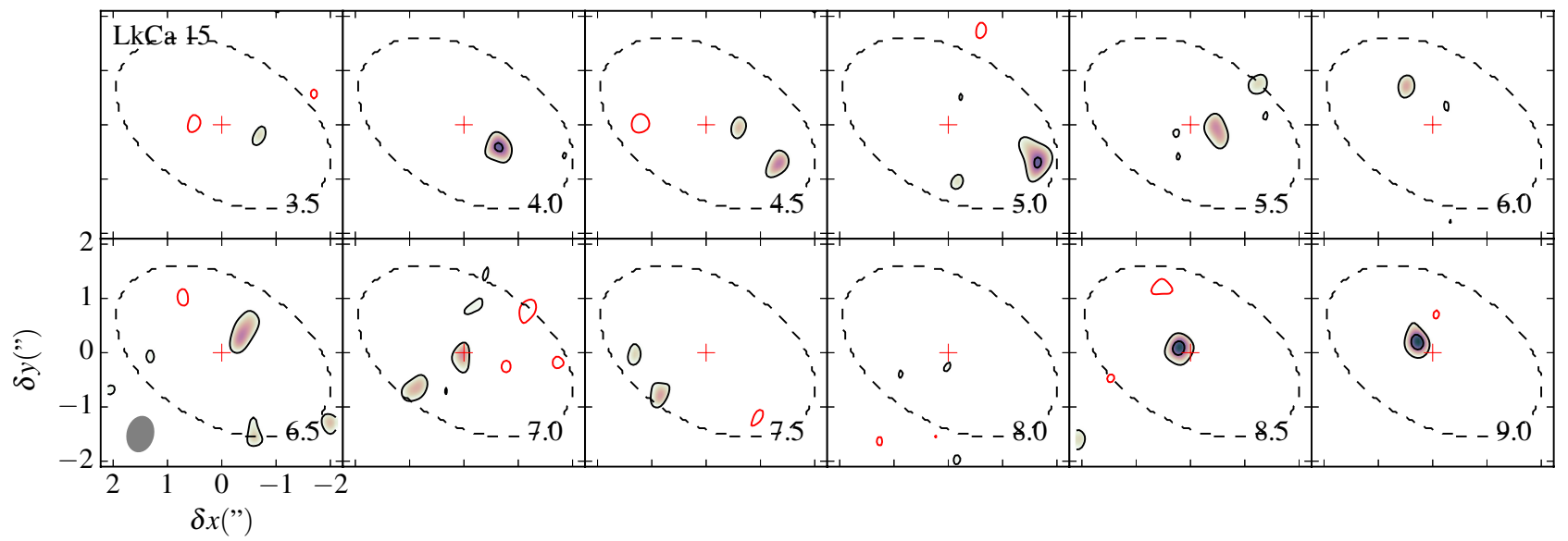

(a) $\mathrm{H}^{13} \mathrm{CN}$

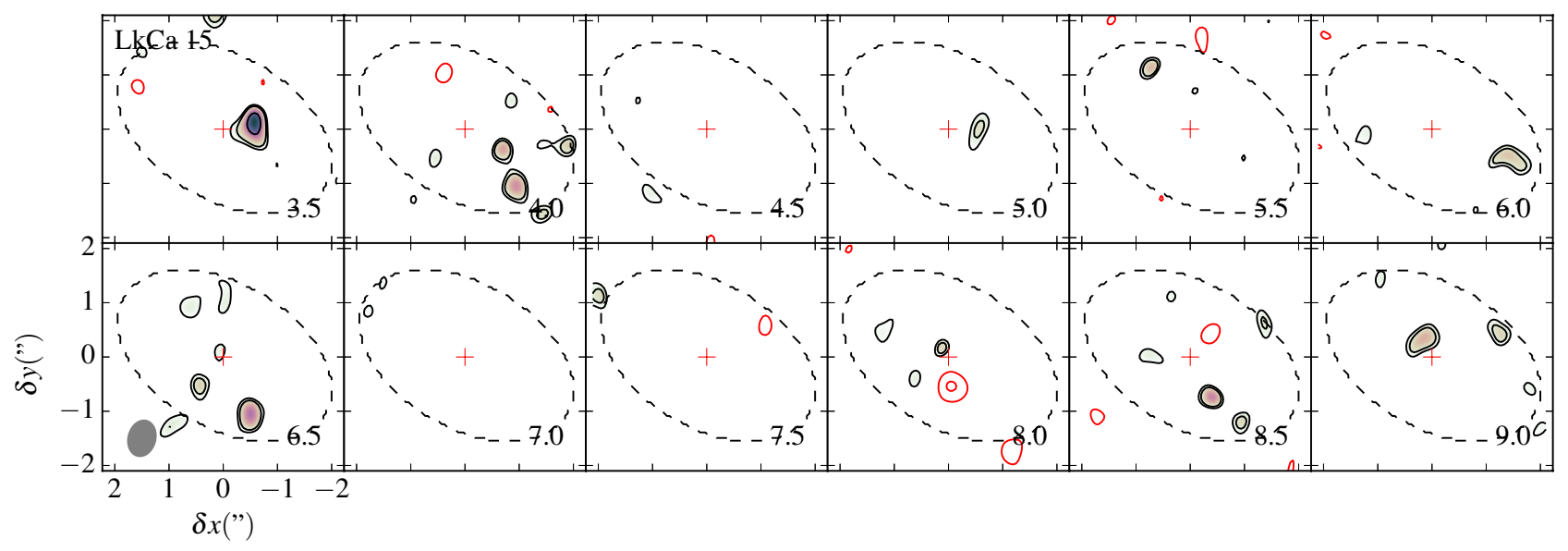

(b) $\mathrm{HC}^{15} \mathrm{~N}$

Figure A.3. : Same as in Fig. A.1 but for LkCa 15. 


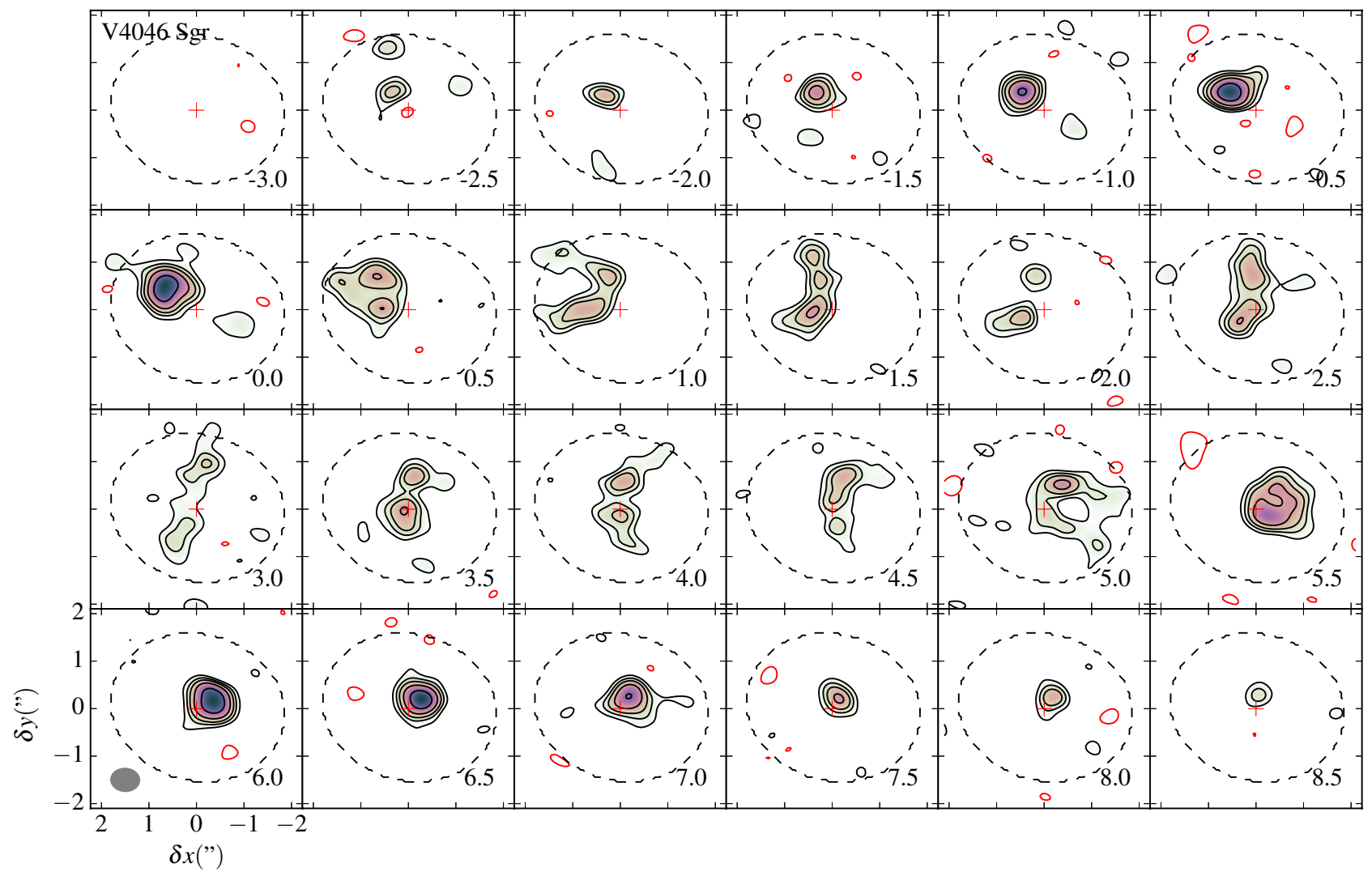

(a) $\mathrm{H}^{13} \mathrm{CN}$

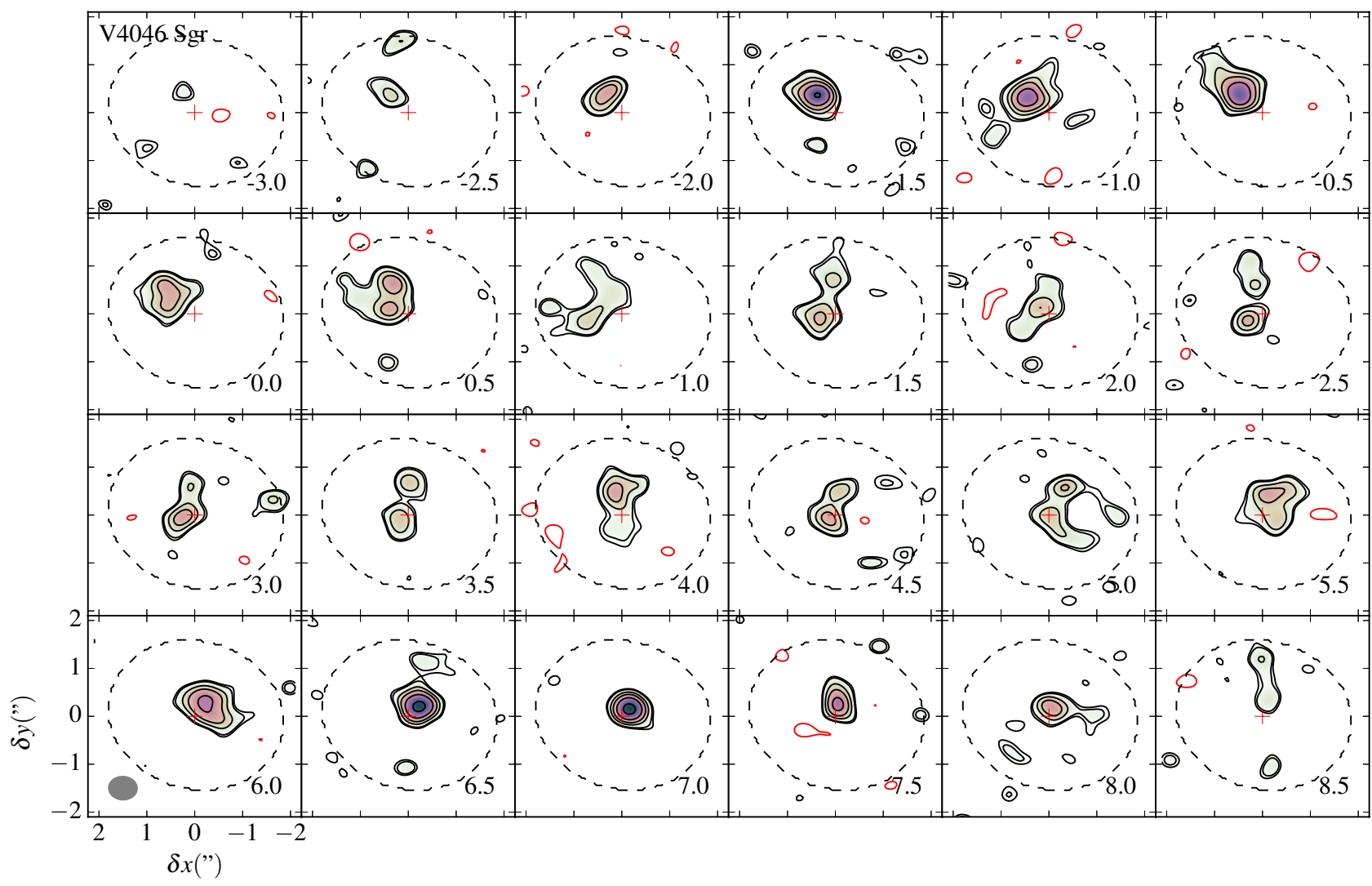

(b) $\mathrm{HC}^{15} \mathrm{~N}$

Figure A.4. : Same as in Fig. A.1 but for V4046 Sgr. 


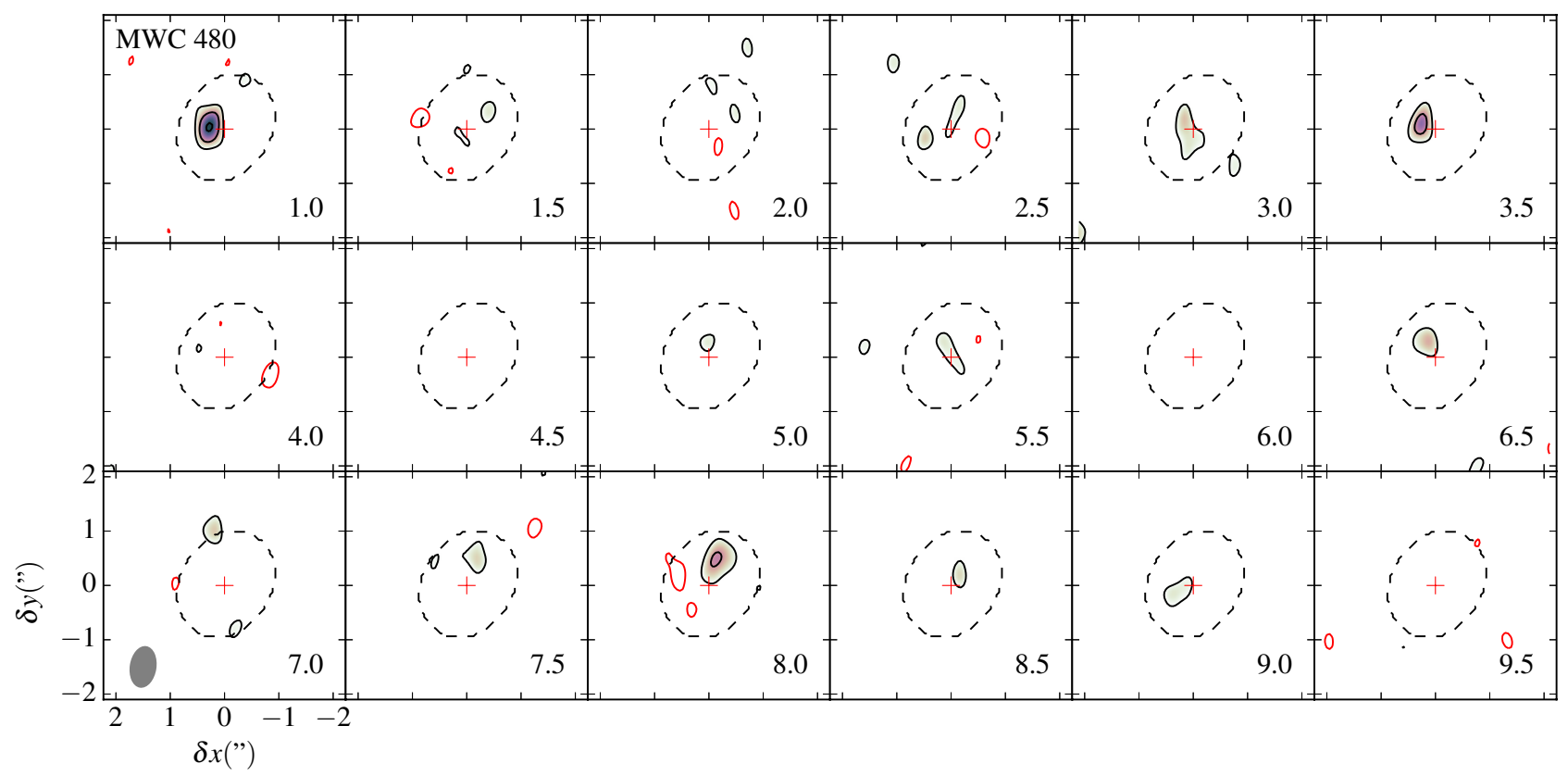

(a) $\mathrm{H}^{13} \mathrm{CN}$

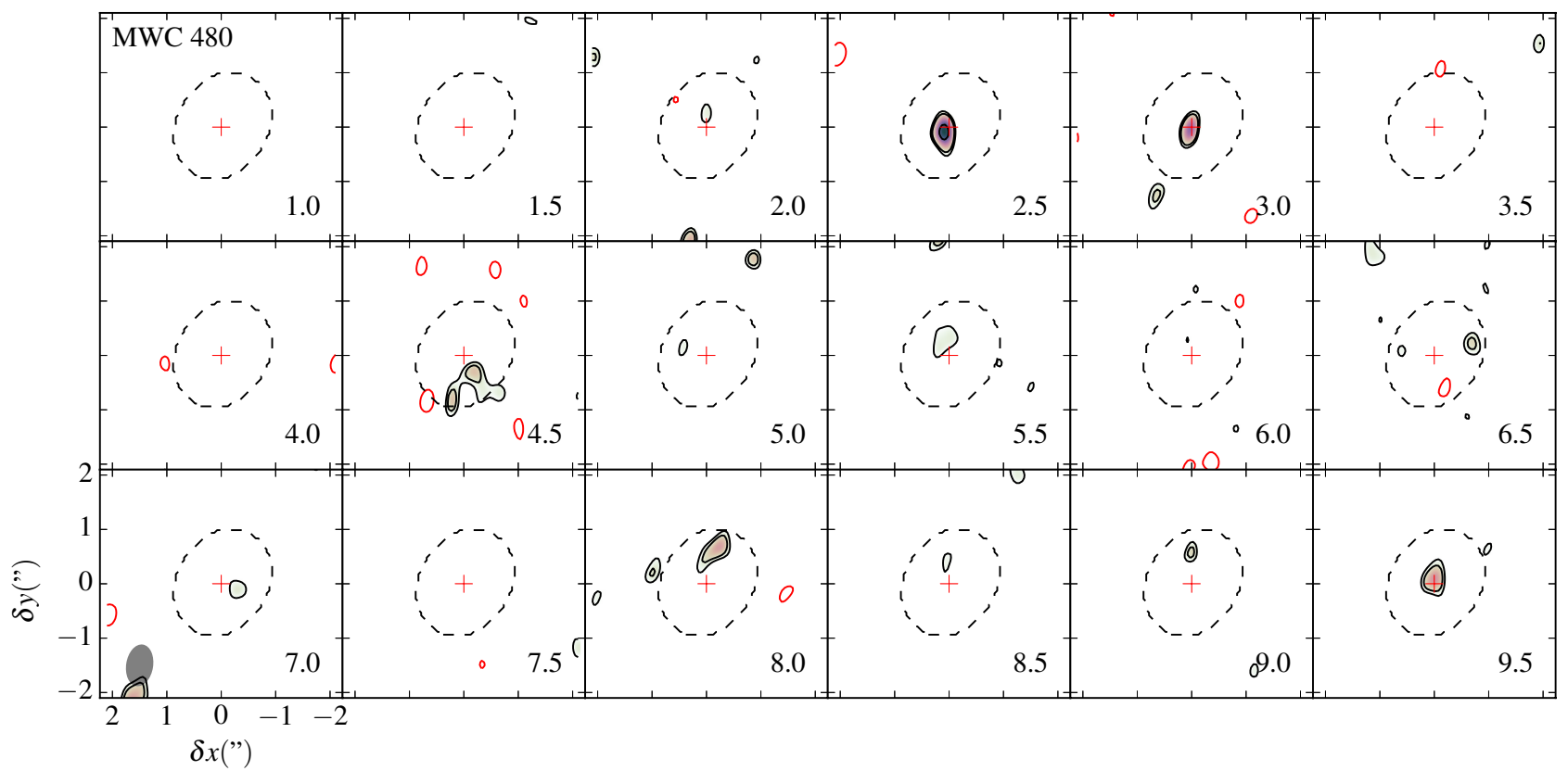

(b) $\mathrm{HC}^{15} \mathrm{~N}$

Figure A.5. : Same as in Fig. A.1 but for MWC 480. 


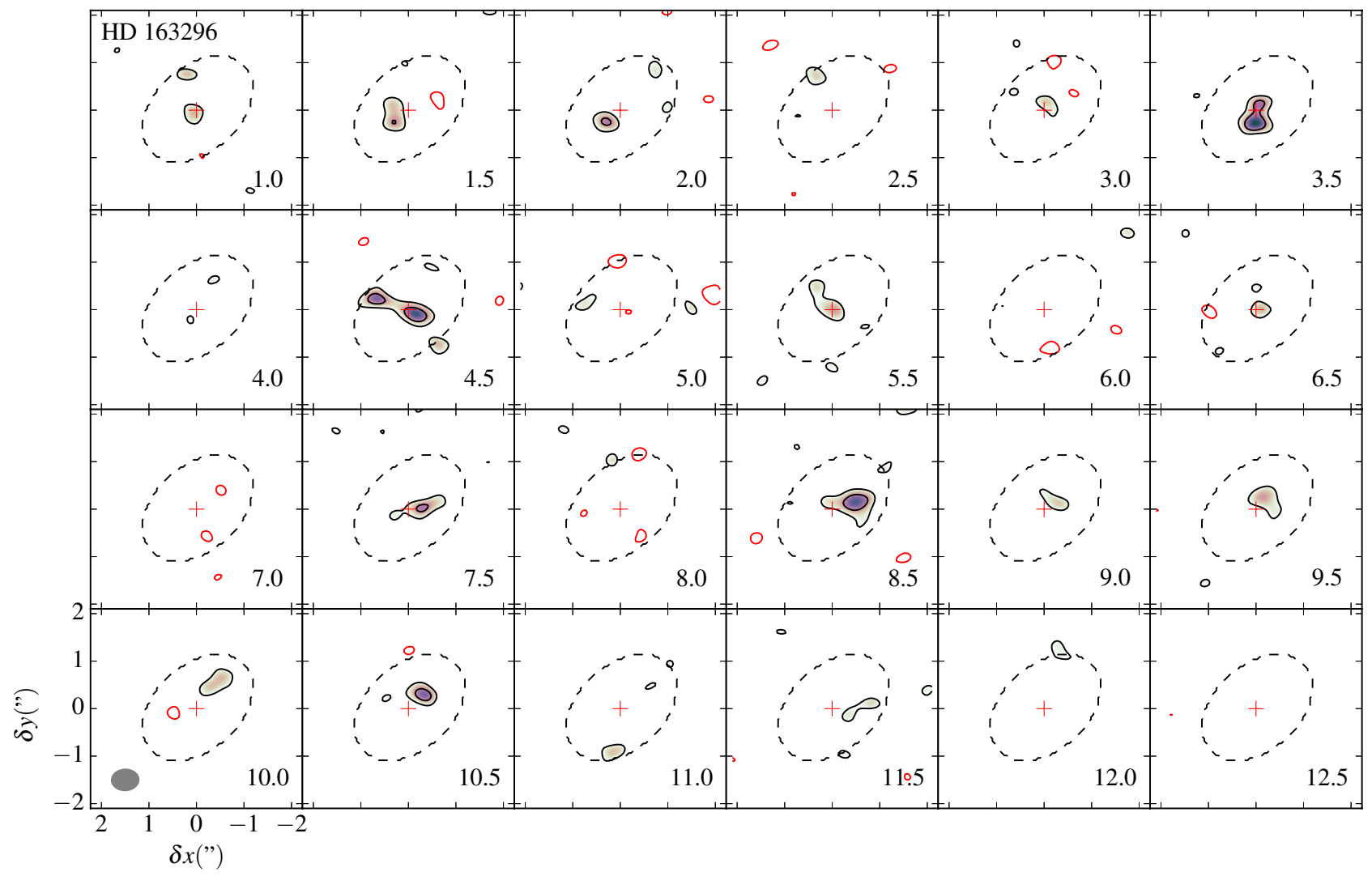

(a) $\mathrm{H}^{13} \mathrm{CN}$

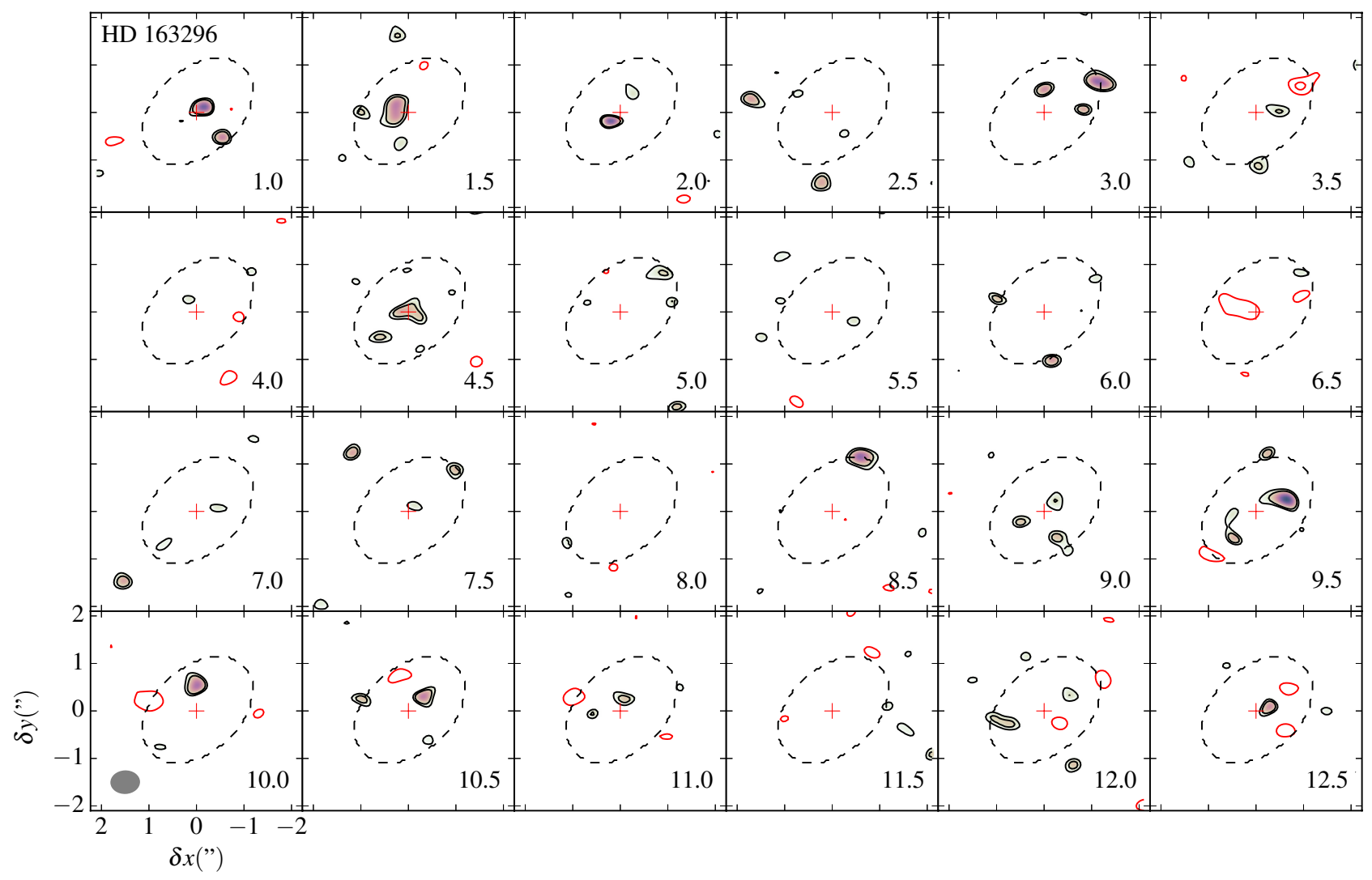

(b) $\mathrm{HC}^{15} \mathrm{~N}$

Figure A.6. : Same as in Fig. A.1 but for HD 163296. 